\title{
Specific Sites within the Ligand-Binding Domain and Ion Channel Linkers Modulate NMDA Receptor Gating
}

\author{
Iehab Talukder, ${ }^{1}$ Priya Borker, ${ }^{2}$ and Lonnie P. Wollmuth ${ }^{2}$ \\ ${ }^{1}$ Graduate Program in Neuroscience and ${ }^{2}$ Department of Neurobiology and Behavior, State University of New York at Stony Brook, Stony Brook, New York \\ $11794-5230$
}

Gating in the NMDA receptor is initiated in the extracellular ligand-binding domain (LBD) and is ultimately propagated via three linkers-S1-M1, M3-S2, and S2-M4 - to the ion channel. M3-S2 directly couples LBD movements into channel gating, but the functional and structural contributions of S1-M1 and S2-M4 to the overall gating process are unknown. A scan of substituted cysteines in and around the NMDA receptor S1-M1 and S2-M4 with a bulky cysteine-reactive reagent identified numerous positions that showed potentiation of glutamate-activated as well as leak currents. As indexed by MK801 (dizocilpine hydrogen maleate), an open channel blocker, this potentiation was attributable to an increase in open probability, an interpretation confirmed for a subset of positions with single-channel recordings. The magnitude of this gating effect, acting through S1-M1 or S2-M4, was dependent on the intrinsic gating properties of the NMDA receptors, being more effective in the inherently low open probability GluN2C- than the higher open probability GluN2A-subunit-containing receptors. For the majority of these potentiation positions, we propose that alteration of gating arises from steric destabilization of contact interfaces where close apposition of the contacting partners is necessary for efficient channel closure. Our results therefore indicate that the NMDA receptor S1-M1 and S2-M4 linkers are dynamic during gating and can modulate the overall energetics of this process. Furthermore, the results conceptualize a mechanistic, as well as a possible structural, framework for pharmacologically targeting the linkers through noncompetitive and subunit-specific modes of action.

\section{Introduction}

NMDA receptors (NMDARs), along with AMPA and kainate receptors, are ion channels gated by glutamate, the major excitatory neurotransmitter in the mammalian CNS. Glutamate-mediated signaling is essential for basic neuron-to-neuron signaling and higher order nervous system processes including learning, memory, and pain perception (Cull-Candy and Leszkiewicz, 2004; Südhof and Malenka, 2008). When aberrant, glutamate-mediated signaling, particularly involving the NMDAR, can trigger and/or complicate numerous acute (e.g., stroke and epilepsy) and chronic (e.g., Alzheimer's and Parkinson's) disease states, as well as psychiatric conditions (e.g., schizophrenia) (Krystal et al., 2003; Waxman and Lynch, 2005).

A hallmark of NMDAR function is gating - the coupling of ligand binding/unbinding to ion channel opening/closing. The core structural components of this process are the extracellular ligand-binding domain (LBD) (S1 and S2) and the channelforming transmembrane domain (M1-M4) (see Fig. 1) (Mayer,

Received 0ct. 30, 2009; revised June 17, 2010; accepted July 14, 2010.

This work was supported by National Institutes of Health-National Institute of Mental Health Grant R01 MH066892 (L.P.W.), an American Heart Association Predoctoral Fellowship (I.T.), and Howard Hughes Medical Institute Grant 52005887 and Undergraduate Research and Creative Activities fellowships (P.B.). We thank Martin Prieto and Catherine Salussolia for helpful discussions and/or comments on this manuscript.

Correspondence should be addressed to Dr. Lonnie P. Wollmuth, Department of Neurobiology and Behavior, Center for Molecular Medicine, State University of New York at Stony Brook, Stony Brook, NY 11794-5230. E-mail: Iwollmuth@notes.cc.sunysb.edu.

DOI:10.1523/JNEUROSCI.5382-09.2010

Copyright $\odot 2010$ the authors $\quad 0270-6474 / 10 / 3011792-13 \$ 15.00 / 0$
2005; Oswald et al., 2007; Sobolevsky et al., 2009). Three peptide linkers-S1-M1, M3-S2, and S2-M4-couple the ligandbinding and transmembrane domains (see Fig. 1). M3-S2, by connecting to the pore-lining and major transmembrane gating element M3, is central to gating (Jones et al., 2002; Sobolevsky et al., 2002; Chang and Kuo, 2008). However, the peripheral S1-M1 and S2-M4, through their interactions with M3-S2 and other proximal structures, may modulate the gating process (Krupp et al., 1998; Villarroel et al., 1998; Yelshansky et al., 2004; Schmid et al., 2007). As such, these linkers represent unexplored drug targets in which receptor activity can be regulated in a noncompetitive manner (Balannik et al., 2005), separate from the mostly failed approaches of competitive and allosteric modulators and pore blockers (Lipton, 2006; Kalia et al., 2008).

A functional NMDAR has four subunits, typically two glycine-binding GluN1s and two glutamate-binding GluN2s. GluN2 has four isoforms (A-D), each imparting distinct biophysical properties to the intact receptor and having diverse spatiotemporal distributions within the CNS (Cull-Candy and Leszkiewicz, 2004; Paoletti and Neyton, 2007). A recent homotetrameric AMPA receptor (AMPAR) structure [Protein Data Bank (PDB) ID 3KG2] revealed the general arrangement and conformations of the LBD-TM linkers. Notably, the linkers were shown to mediate the symmetry mismatch between the extracellular (twofold) and transmembrane (fourfold) domains by taking on two different conformations. In the NMDAR, the two conformations are apparently subunit specific (Sobolevsky et al., 2009). Nevertheless, there are limitations in relating specific linker po- 
sitions in this AMPAR structure to their NMDAR counterparts because of variability in their lengths, amino acid composition, and low resolution of certain linkers in the structure (supplemental Fig. 1, available at www.jneurosci.org as supplemental material). In addition, a single structural snapshot does not identify functionally important elements of gating.

Taking advantage of substituted cysteines, we identify specific positions encompassing the NMDAR S1-M1 and S2-M4 that can modulate gating. This modulation is subunit specific, being dependent on the inherent gating properties as determined by the expressed GluN2 isoform (GluN2C or GluN2A). We propose that most of these positions are located at sites of transient gatingdependent interactions whose stabilization or destabilization can shift overall gating energetics toward the channel-open or -closed states, respectively.

\section{Materials and Methods}

\section{Materials}

Methanethiosulfonate (MTS) reagents, 3-(triethylammonium)propyl methanethiosulfonate bromide (PTrEA), [2-(trimethylammonium) ethyl]methanethiosulfonate bromide (MTSET), and 2-aminoethyl methanethiosulfonate hydrobromide (MTSEA), were purchased from Toronto Research Chemicals. The GluN1 glycine site antagonist, 5,7-dichlorokynurenic acid (DCKA), was purchased from Tocris Bioscience. The GluN2 glutamate site antagonist, DL-2-amino-phosphonopentanoic acid (APV), and the NMDA receptor channel blocker, dizocilpine hydrogen maleate (MK801), were purchased from Sigma-Aldrich.

\section{Mutagenesis and expression}

Cysteine substitutions in the GluN1 (K526-L533 in S1-M1 and S782-A888 in S2-M4) and GluN2C (R518-P525 in S1-M1 and N781-S789 in S2-M4) subunits were generated using PCR-based methods. Various cysteinesubstituted GluN1 and GluN2C subunits were made previously: GluN1 positions D534-L544 (S1-M1), A635-R641 (M3-S2), and T789-A796 (S2-M4) (Beck et al., 1999); GluN2C positions P642-R645 (M3-S2) (Watanabe et al., 2002); and GluN2C positions S526-W537 (S1-M1), A630-V641 (M3-S2), and K790-G798 (S2-M4) (Sobolevsky et al., 2007). Mutations were initially made in clones present in a pSp64T-derived vector. Subsequently, a fragment encompassing the mutation was subcloned back into the wild-type template. All constructs were sequenced over the entire length of the replaced fragment. cRNA was transcribed for each expression construct using SP6 RNA polymerase (Ambion). The cRNA was examined electrophoretically on a denaturing agarose gel. Dilutions of RNA $(0.01-0.1 \mu \mathrm{g} / \mu \mathrm{l})$ were prepared to achieve optimal expression. Wild-type and mutant GluN1 and GluN2C subunits were coexpressed in Xenopus laevis oocytes. Oocytes were prepared, injected, and maintained as previously described (Sobolevsky et al., 2002). Recordings were made 2-5 d after injections.

In GluN2A, an endogenous cysteine (C399) reacts with MTS reagents resulting in $\sim 30 \%$ inhibition of current amplitudes. Therefore, all of our studies involving GluN2A were done using a GluN2A construct in which this cysteine was mutated to an alanine (C399A) (Choi et al., 2001), referred to here as GluN2A'.

\section{Whole-cell current recordings and data analysis}

Whole-cell currents of Xenopus oocytes were recorded at room temperature $\left(20^{\circ} \mathrm{C}\right)$ using two microelectrode voltage clamp (TEVC) (DAGAN TEV-200A; Dagan) with Cell Works software (NPI Electronic). Microelectrodes were filled with $3 \mathrm{M} \mathrm{KCl}$ and had resistances of 1-4 M $\Omega$. To maximize solution exchange rates, we used a narrow flow-through recording chamber with a small volume of $\sim 70 \mu$ l. When recording GluN1-GluN2C, the external solution consisted of the following (in mM): $115 \mathrm{NaCl}, 2.5 \mathrm{KCl}, 0.18 \mathrm{CaCl}_{2}$, and 5 HEPES, pH 7.2, $\mathrm{NaOH}$, unless otherwise noted. When recording GluN1-GluN2A', $\mathrm{BaCl}_{2}$ was substituted for $\mathrm{CaCl}_{2}$ (to prevent $\mathrm{Ca}^{2+}$-dependent desensitization) and 100 $\mu \mathrm{M}$ EDTA (to minimize $\mathrm{Zn}^{2+}$-mediated modification) was added to the external solution. All reagents, including glutamate (200 $\mu \mathrm{M})$, glycine (20
A

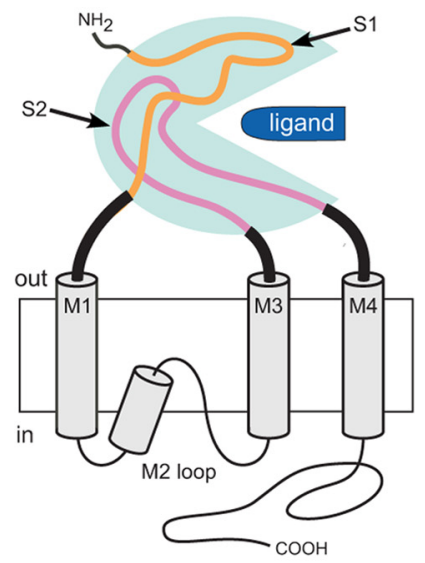

C GluN1 GluN2C

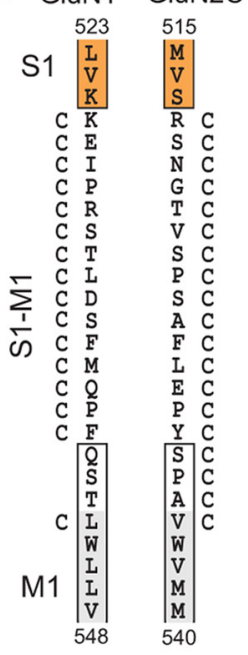

B

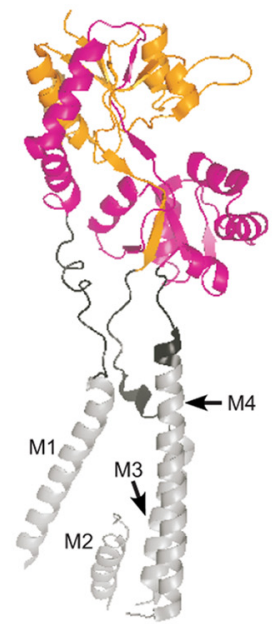

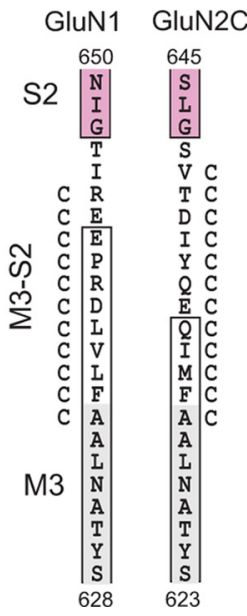

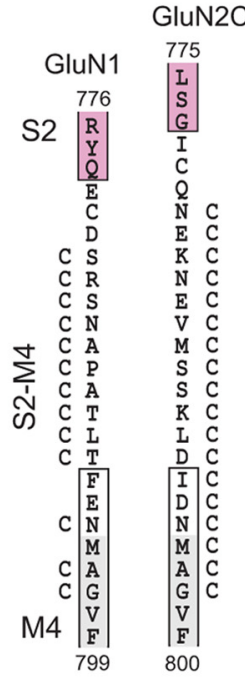

Figure 1. The linkers S1-M1, M3-S2, and S2-M4 connect the extracellullar LBD to the transmembrane segments in a glutamate receptor subunit. $A, S$ chematic representation of the functional core of an ionotropic glutamate receptor (iGluR) subunit. The extracellular LBD, shown in cyan, is composed of lobes $\mathrm{S} 1$ (shown in orange) ( $\mathrm{N}$-terminal to M1) and $\mathrm{S} 2$ (shown in magenta) (between M3 and M4). The hydrophobic segments M1, M3, and M4, as well as the M2 pore loop, are shown as gray cylinders. The thick black bars indicate regions in and around the linkers S1-M1, M3-S2, and S2-M4, in which cysteines were substituted. $\boldsymbol{B}$, Ribbon representation of a

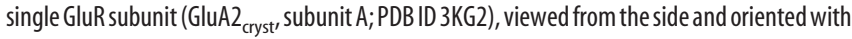
the extracellular solution above. The LBD, the linkers, and the transmembrane segments are shown with colors as in $\boldsymbol{A}$. C, Sequence of the regions encompassing the three linkers in the GluN1 and GluN2C subunits. The proximal parts of the presumed hydrophobic segments M1, M3, and M4 (Schmid et al., 2007) are highlighted in gray. The boxed regions around these hydrophobic segments show the proximal $\alpha$-helical extent of the transmembrane segments as revealed by GluA2 $_{\text {cryst }}$ (Sobolevsky et al., 2009). GluN1 is assumed to take the A/C conformation, whereas GluN2C, the B/D conformation. The proximal parts of $S 1$ and $S 2$ are highlighted in orange and magenta, respectively. Positions substituted with cysteines and tested for PTrEA reactivity are indicated (see Materials and Methods). Certain positions, specifically those without an adjacent " $\mathrm{C}$," were not tested because MTS reagents did not persistently alter their current amplitudes (Beck et al., 1999). Numbering is for the mature protein.

$\mu \mathrm{M})$, APV $(100 \mu \mathrm{M})$, DCKA $(10 \mu \mathrm{M})$, and MTS reagents, were applied with the bath solution.

Data analysis was done using Igor Pro (Wavemetrics) and Microsoft Excel. For analysis and illustration, leak currents were subtracted from total currents. Results are presented as mean \pm SEM $(n \geq 4)$. An ANOVA or Student's $t$ test was used to define statistical differences. 
The Dunnet test was used for multiple comparisons. Significance was assumed to be $p<$ 0.05 .

\section{Experimental protocols}

NMDA receptor cysteine-substituted mutant channels were probed from the extracellular side with MTS reagents. MTS reagentcontaining solutions were prepared, stored, and applied as previously described (Sobolevsky et al., 2002).

Steady-state reactions. Steady-state reactions were quantified at a holding potential of -60 $\mathrm{mV}$ (see Fig. 2A-D). Baseline glutamateactivated current amplitudes $\left(I_{\text {pre }}\right)$ were established by three to five $15 \mathrm{~s}$ applications of glutamate and glycine. All agonist or any other reagent applications were separated by 30 to $120 \mathrm{~s}$ washes in glutamate-free solution. PTrEA $(1 \mathrm{~mm})$ was applied for $60 \mathrm{~s}$ either in the presence of agonists or in their absence (but in the presence of the competitive antagonists APV and DCKA). After the PTrEA exposure, current amplitudes $\left(I_{\text {post }}\right)$ were determined again using three to five agonist applications. MTSET (1 mM) or MTSEA (1 mM) was applied only in the presence of agonists. The change in glutamate-activated current amplitude, expressed as a percentage (percentage change), was calculated as follows: $=\left(1-I_{\text {post }} / I_{\text {pre }}\right) \times$ 100 . In certain instances, we corrected for observed current rundown by fitting a single exponential function to a minimum of three pre-MTS reagent glutamate-activated current amplitudes.

MK801 inhibition. MK801 is an irreversible (on the timescale of tens of minutes) open channel blocker at hyperpolarized potentials (Huettner and Bean, 1988), and correspondingly its rate/extent of block has been used previously to index $P_{\mathrm{o}}$ (Huettner and Bean, 1988; Jahr, 1992; Rosenmund et al., 1995; Chen et al., 1999). We quantified the rate of MK801 inhibition of open channels using the protocol shown in Figure 6, $A$ and B. A continuous MK801 application in the presence of agonists was started after glutamate-activated currents had reached steady-state (typically 15-30 s) or after PTrEA had potentiated glutamate-activated currents to steady-state levels (typically 45-75 s). Through an initial trial-and-error process for each cysteine-substituted receptor, we adjusted the concentration of MK801 to reach maximal block within 4-10 min: $300 \mathrm{~nm}$ for GluN2C(A630C), $200 \mathrm{~nm}$ for GluN1 (P787C), $150 \mathrm{~nm}$ for GluN2C(E635C), $25 \mathrm{~nm}$ for GluN2C(S533C), and $50 \mathrm{~nm}$ for the remaining cysteine-substituted receptors. Resulting changes in current amplitude were fitted with a single-exponential function. The reciprocal of the time constant $(\tau)$ of this fit multiplied by the concentration of MK801 defined the apparent second-order rate constant for block: $k=1 /(\tau[\mathrm{MK} 801])$. We used the rate of MK801 wholecell current inhibition to index relative $P_{\mathrm{o}}$, not an absolute $P_{\mathrm{o}}$, which could be complicated since, in addition to open channel block, MK801 might also interact with the gating machinery (Dilmore and Johnson, 1998). Moreover, for GluN1-GluN2C, (+)MK801 (the stereoisomer we use) does not have the paradoxical $\mathrm{pH}$ dependence of increasing the affinity of channel block despite lowering $P_{\mathrm{o}}$ that (-)MK801 exhibits (Dravid et al., 2007).

Reaction rates. Reaction rates in the presence of glutamate and glycine $\left(k_{+}\right)$and in their absence but in the presence of APV and DCKA $\left(k_{-}\right)$ were determined using "pulsive" protocols (see Fig. $9 A, B$ ), as described in detail by Sobolevsky et al. (2002). Changes in current amplitude were fitted with a single exponential. The reciprocal of the time constant $(\tau)$ of this fit multiplied by the concentration of the MTS reagent defined the apparent second-order rate constant for chemical modification $k_{+}$or $k_{-}=1 /(\tau[\mathrm{C}])$.
B

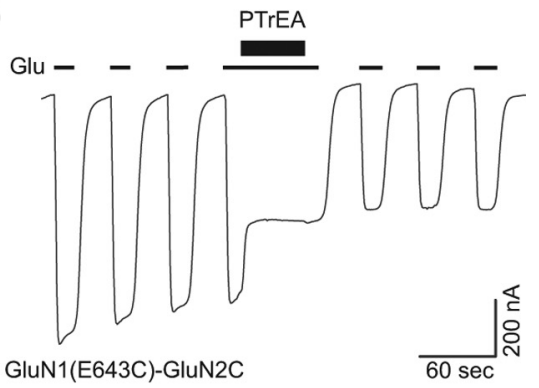

D

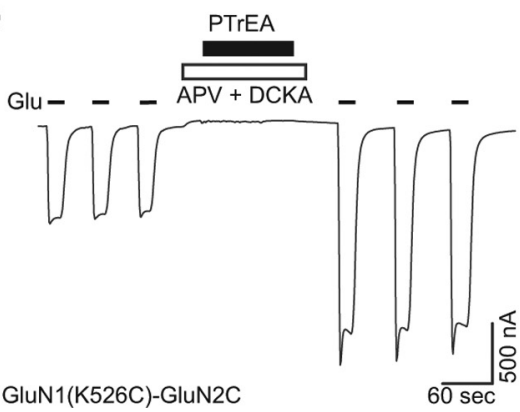

Figure 2. Steady-state recording protocols to assay accessibility of substituted cysteines to PTrEA. Examples of whole-cell PTrEA produ $\left(\begin{array}{l}\text { pre } \\ \text { post }\end{array}\right.$ certain mutants, leak current was also significantly changed after PTrEA application (C).

\section{Single-channel recordings and analysis}

Before recording, oocytes were bathed in a hypertonic solution consisting of the following (in mM): $200 \mathrm{~K}$-aspartate, $20 \mathrm{KCl}, 5 \mathrm{EGTA}$, and 10 HEPES, pH 7.4, KOH, for 5-15 min, and then manually devitellinized. Oocytes were then placed in the recording chamber under the continuous flow of a bathing solution (in mM: $115 \mathrm{NaCl}, 2.5 \mathrm{KCl}, 0.50 \mathrm{CaCl}_{2}, 1$ $\mathrm{MgCl}_{2}$, and 5 HEPES, $\left.\mathrm{pH} 7.2, \mathrm{NaOH}\right)$. Currents in outside-out patches, isolated from these oocytes, were recorded at room temperature (20$23^{\circ} \mathrm{C}$ ). The recording external solution, which was applied using a single barrel application pipette, was the same as the bathing solution but without added $\mathrm{MgCl}_{2}$; to this solution, we added $0.5 \mathrm{~mm}$ glycine and $1 \mathrm{~mm}$ glutamate, $\mathrm{pH} 7.2, \mathrm{NaOH}$, with or without PTrEA (1 mM). The internal pipette solution contained the following (in $\mathrm{mm}$ ): $10 \mathrm{KCl}, 90$ K-gluconate, 2 MgATP, 5 phosphocreatine, 1 BAPTA, and 10 HEPES, $\mathrm{pH} 7.4, \mathrm{KOH}$.

Microscopic currents were recorded using an Axopatch 200B amplifier (Molecular Devices) with PatchMaster software (HEKA Elektronik) and were initially filtered at $10 \mathrm{kHz}$ using a four pole low-pass Bessel filter and digitized at $50 \mathrm{kHz}$. All recordings were made at $-80 \mathrm{mV}$. Pipettes were pulled from thick-walled borosilicate glass, coated with Sylgard (Dow Corning), and fire polished immediately before use. Pipette resistances were 5-25 M $\Omega$ when filled with the pipette solution and measured in the bathing solution. The pipette holder was cleaned daily with $70 \%$ ethanol and stored in Drierite overnight. The level of the batch solution was kept as low as possible. A typical experiment consisted of at least three continuous control pre-PTrEA $30 \mathrm{~s}$ applications of agonists, followed by a $1 \mathrm{~min}$ exposure to agonists plus PTrEA ( $1 \mathrm{~mm}$ ), and then back to continuous agonist application until the patch became unstable. Although patches certainly contained multiple channels (the exact number was unknown but presumably was between 1 and 8), GluN1-GluN2C receptors have a very low $P_{\mathrm{o}}(0.01)$ (Dravid et al., 2008), making double openings extremely rare ( $<2 \%$ of the time). If double openings were more prominent in the baseline recording, we terminated the experiment. 


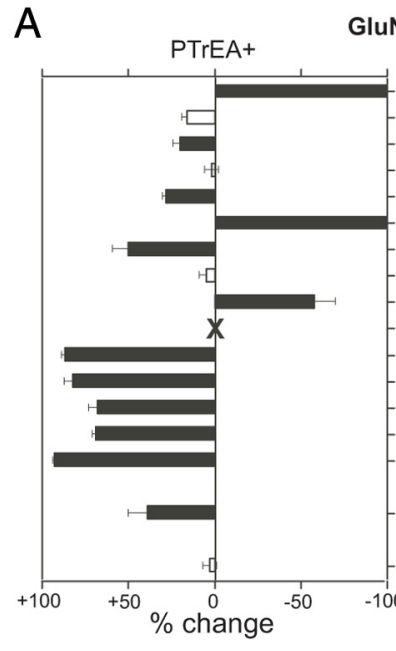

GluN1 S1-M1

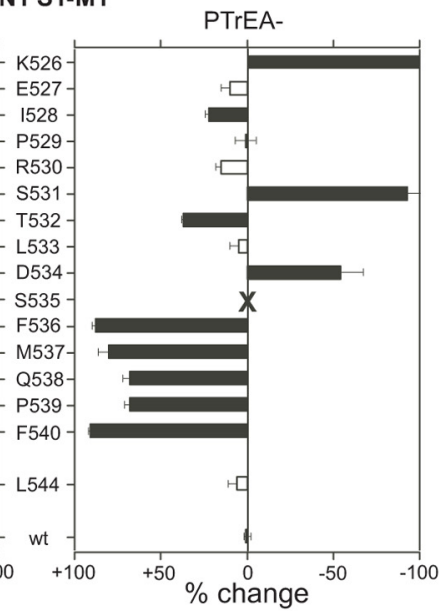

B

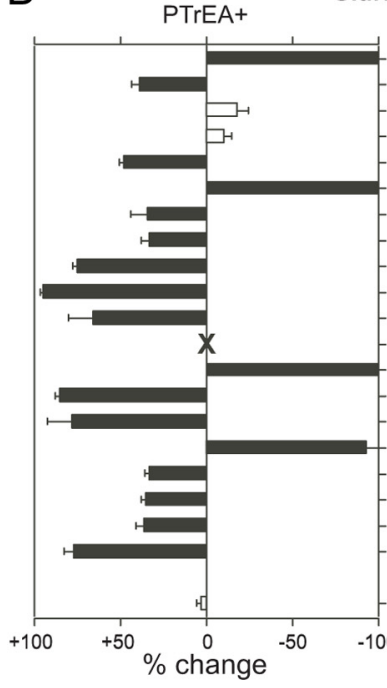

GluN2C S1-M1

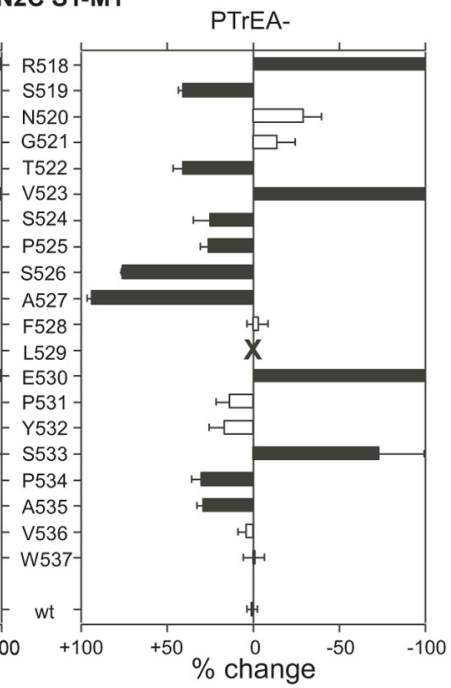

Figure 3. Effects of PTrEA on substituted cysteines in and around the GluN1 and GluN2CS1-M1 linkers. $A, B$, Mean percentage change ( \pm 2 SEM) in glutamate-activated current amplitudes measured before $\left(I_{\text {pre }}\right)$ and after $\left(I_{\text {post }}\right)$ exposure of wild-type and cysteine-substituted GluN1 $(\boldsymbol{A})$ or GluN2C $(\boldsymbol{B})$ subunits to PTrEA in the continuous presence (PTrEA + , left) or absence (PTrEA- , right) of agonists. The left and right pointing bars indicate inhibition and potentiation, respectively. For positions with percentage change $=-100$, potentiation was stronger than $100 \%$. The filled bars indicate that the value of percentage change is statistically different from that of wild-type GluN1-GluN2C $(p<0.05)$. C, Summary of discrete effects of PTrEA, applied in the presence of agonists, on substituted cysteines in and around the GluN1 and GluN2C S1-M1 linkers. The adjacent circles indicate that positions were tested for reactivity with PTrEA: unfilled circles, no effect on glutamate-activated current; black circles, current inhibition; red circles, current potentiation. X, No detectable glutamate-activated current; green highlight, significant PTrEA-induced change in leak current.

Currents were exported from PatchMaster to Igor Pro for initial processing including subtraction of mean baseline current, resampling, and additional filtering. Records were then exported from Igor Pro to QuB (www.qub.buffalo.

edu) for idealization. Idealized records were then exported back to Igor Pro and/or ChannelLab (Synaptosoft) for additional analysis.

Records were idealized using QuB. Currents were resampled at $25 \mathrm{kHz}$ and digitally refiltered at $5 \mathrm{kHz}$ to a final frequency of $\approx 4.5 \mathrm{kHz}$. Idealization was done, with an imposed "dead time" of $40 \mu$ s (resolution of 80 $\mu$ s), using the SKM method (Qin, 2004) with a model that had two open classes and one closed class (Stern et al., 1992; Dravid et al., 2008), all interconnected. The amplitude and SD for the single closed state (baseline) was defined using the "grab" function in QuB. We initially also used the "grab" function to estimate amplitudes of the two open classes for wild-type and mutant receptors. The current amplitudes were qualitatively comparable. Nevertheless, to reduce variability because of the specific starting values "grabed," we set the current amplitudes of the two

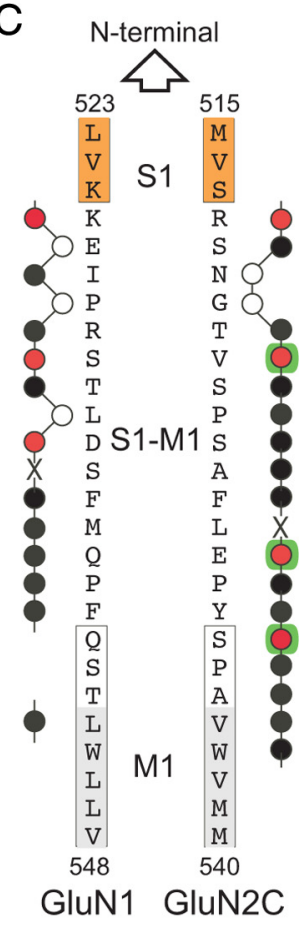

open classes to -3.2 and $-1.8 \mathrm{pA}$, based on previously published values for single-channel conductance sublevels for recombinant GluN1GluN2C receptors expressed in oocytes (Stern et al., 1992). The initial SDs of these open classes were set to that for baseline. We then allowed the mean current levels and SDs to be reestimated during idealization.

Current amplitudes for the two conductance levels (lower and higher) were derived from all-point histograms (QuB). Mean lifetimes were derived from single-exponential fits to dwell time histograms of the identified conductance levels. Two exponentials were fit to dwell time histograms of composite open times (ChannelLab). Patch $P_{\mathrm{o}}$ was determined from the total open time (low plus high conductance) divided by the total time of the recording. We did not correct for missed events, which would be approximately equivalent for both before and after PTrEA.

\section{Results}

To identify key positions in the NMDAR linkers that can influence gating, we took advantage of substituted cysteines in and around the GluN1 and GluN2C linkers (Fig. 1C). We screened these substituted cysteines, one at a time, with the cysteinereactive reagent PTrEA, anticipating that, given its positive valence and large size, it might in certain instances affect gating movements of the linkers.

\section{Accessibility scan of the GluN1 and GluN2C linkers}

The protocol used to determine the effect of PTrEA on whole-cell glycineand glutamate-activated (referred to as glutamate-activated) current amplitudes for cysteine-substituted NMDARs is illustrated in Figure 2. We compared current amplitudes before $\left(I_{\text {pre }}\right)$ and after $\left(I_{\text {post }}\right)$ exposure to extracellular PTrEA (1 mM, thick lines) applied in the continuous presence of agonists (Fig. $2 A-C$ ). We also determined PTrEA accessibility in the absence of agonist, but in the presence of the competitive antagonists DCKA (at the GluN1 site) and APV (at the GluN2 site) to minimize channel opening (Fig. 2D). Since NMDARs composed of GluN1 and GluN2C subunits do not undergo any apparent desensitization (Krupp et al., 1996), we assume that, during PTrEA application, the cysteine-substituted GluN1-GluN2C receptors exist primarily in the open and closed states (in the presence of agonists) or in the closed state (in the absence of agonists but presence of antagonists). Similar exposures of wild-type GluN1-GluN2C receptors to PTrEA produced no significant changes in current amplitudes indicating that possible modifications of endogenous cysteines do not affect receptor function.

In terms of whole-cell glutamate-activated currents, three distinct outcomes were observed after PTrEA exposure to cysteinesubstituted GluN1-GluN2C receptors: no significant change (Fig. $2 A$ ), inhibition (Fig. $2 B$ ), or potentiation (Fig. 2C,D). Figures 3-5 summarize the mean percentage change (percentage 
change) in glutamate-activated current amplitudes of wild-type and mutant GluN1GluN2C receptors containing substituted cysteines in and around S1-M1 (Fig. 3), M3-S2 (Fig. 4), or S2-M4 (Fig. 5) with PTrEA applied either in the presence (PTrEA+, left) or absence (PTrEA-, right) of agonists. The unfilled bars indicate cysteine-substituted positions where PTrEA application did not significantly change glutamate-activated current amplitudes. The filled bars showing either current inhibition ( + percentage change) or potentiation ( - percentage change) indicate positions where percentage change was significantly different from that observed in wild-type GluN1-GluN2C. GluN1(S535C) and GluN2C(L529C) (Fig. 3A,B), denoted by the symbol $\mathrm{X}$, showed no detectable glutamate-activated currents. Current amplitudes in GluN2C(L791C) (denoted by asterisk in Fig. 5B) never reached steady state (up to 4 min agonist application) (data not shown), but they were clearly altered in response to PTrEA. PTrEA-induced current potentiation for GluN2C(I793C) (denoted by double asterisks in Fig. $5 B$ ) never reached steady state (up to $5 \mathrm{~min}$ and $4 \mathrm{~mm}$ PTrEA application was attempted) (data not shown). Figures $3 C, 4 C$, and $5 C$ show a summary of the effects of PTrEA, when applied in the presence of agonists, on the positions tested (adjacent circle): no effect, open circle; inhibition, black circle; and potentiation, red circle. Positions where leak currents (i.e., currents in the absence of agonists) were significantly altered after PTrEA [Fig. 2C, GluN2C(S789C); supplemental Table 1, available at www.jneurosci.org as supplemental material] are highlighted in green.

\section{Interpretation of PTrEA-induced effects on cysteine- substituted positions}

Because of their extracellular location, the GluN1-GluN2C linkers are widely accessible, with most cysteine-substituted receptors undergoing significant PTrEA-induced changes in glutamate-activated current amplitude (inhibition or potentiation, 68 of 87 positions tested). Additionally, some of the seemingly inaccessible positions (i.e., the white positions [Fig. 4A, B, GluN1(P642C) and GluN2C (I633C), both in M3-S2]) might be accessible, but reaction with PTrEA did not affect glutamate-activated current amplitudes (silent reaction). Overall, the linkers showed limited differences in reactivity when PTrEA was applied either in the presence or absence of agonists. This lack of discrete state dependence probably reflects the extracellular location of the linkers, with no physical barriers (i.e., activation gate, membrane lipids, etc.) preventing global access, but it does not preclude the possibility of local state-dependent restrictions on accessibility below the limits of resolution of the steady-state protocol.

Of the positions that do undergo significant PTrEA-induced changes in glutamate-activated current amplitudes, notable are those that show potentiation after PTrEA (Figs. 3C, 4C, 5C, red positions). Previously, it was proposed, albeit untested, that potentiation after modification by MTS reagents reflects a gating effect with the overall energetics shifted toward channel opening (Yuan et al., 2005; Sobolevsky et al., 2007). Consistent with this idea, 8 of the 18 potentiation positions showed significant PTrEA-induced increases in leak current (supplemental Table 1, available at www.jneurosci.org as supplemental material), suggesting constitutive channel opening after PTrEA (note only 9 total positions showed significant PTrEA-induced increases in leak currents). Surprisingly, the potentiation positions are prominent in S1-M1 and S2-M4-regions typically not associated with channel gating.

To address the possible role of the linkers in gating, we considered the basis for PTrEA-induced changes in current amplitudes by focusing mainly on potentiation positions. Macroscopic whole-cell currents $\left(I_{\mathrm{wc}}\right)$ are defined by the equation, $I_{\mathrm{wc}}=N \gamma P_{\mathrm{o}}$, where $N$ is the number of surface receptors, $\gamma$ is the singlechannel conductance, and $P_{\mathrm{o}}$ is the open probability of the channel. Within the seconds-to-minutes timescale of PTrEA reaction and consequent modification of current amplitudes, it is unlikely that $N$ changes. Hence, a change in whole-cell current after reaction to PTrEA (or any other MTS reagent) is the net effect of changes in $\gamma$ (a conductance effect) and $P_{\mathrm{o}}$ (a gating effect), $\Delta I_{\mathrm{wc}}=\Delta \gamma+\Delta P_{\mathrm{o}}$.

The rate of channel block by MK801 becomes faster in receptors showing PTrEA-induced current potentiation Initially, to test whether an increase in $P_{\mathrm{o}}$ underlies PTrEA-induced current potentiation, we measured rates of whole-cell current inhibition by the mostly irreversible NMDA receptor open channel blocker MK801 (Huettner and Bean, 1988). Since access to the MK801 blocking site in the pore depends on channel opening, the rate of MK801 block in wild-type and cysteine-substituted/ PTrEA-treated receptors should be proportional to $P_{\mathrm{o}}$, assuming 


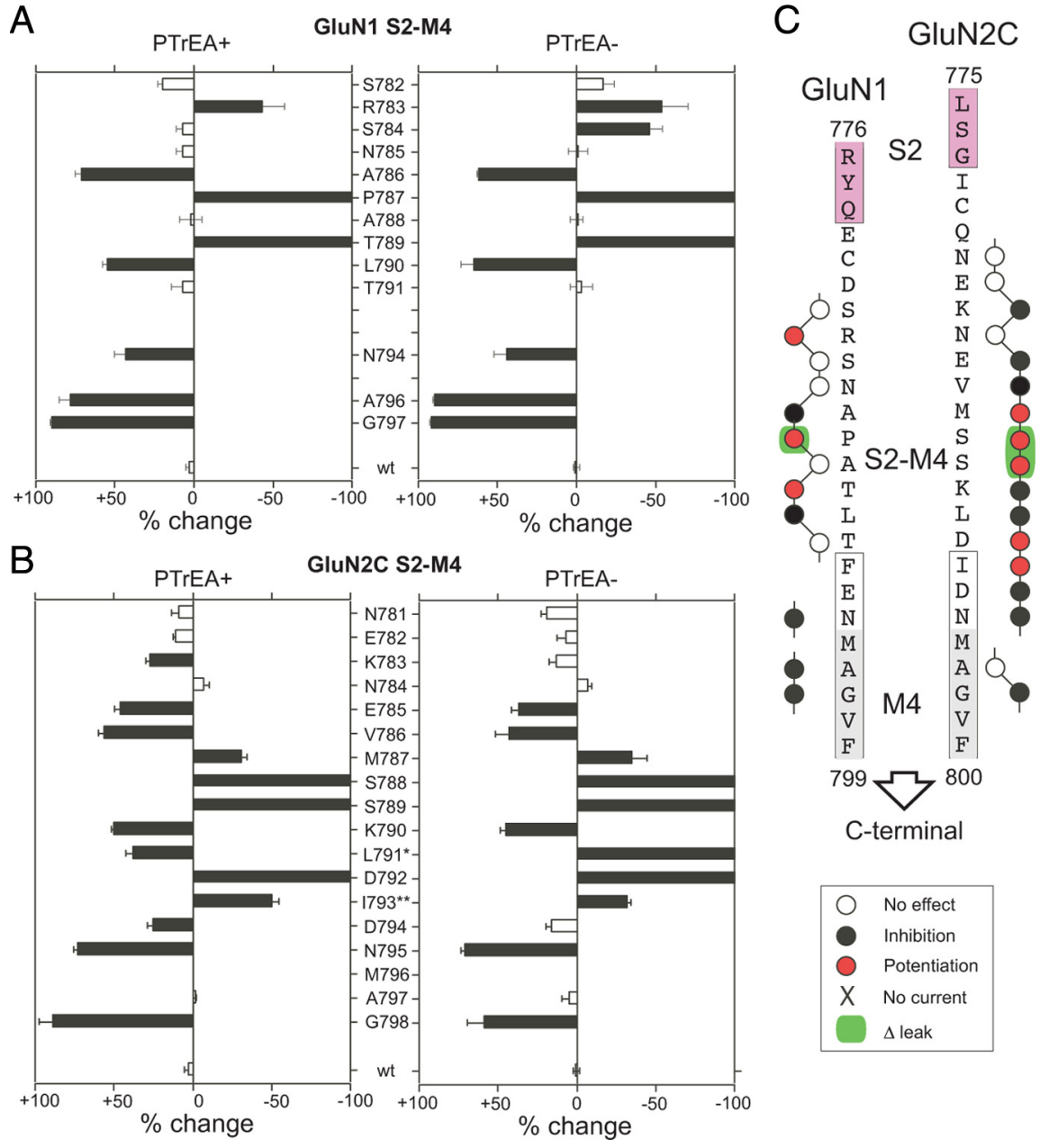

Figure 5. Effects of PTrEA on substituted cysteines in and around the GluN1 and GluN2C S2-M4 linkers. Same as Figure 3 , but for S2-M4.

that the interaction of MK801 with its blocking site is not greatly perturbed (see Materials and Methods) (supplemental Fig. 2, available at www.jneurosci.org as supplemental material).

Figure $6 \mathrm{~A}$ illustrates MK801-mediated inhibition of glutamate-activated whole-cell current in wild-type GluN1GluN2C. NMDAR $P_{\mathrm{o}}$ is highly dependent on the GluN2 subtype (Chen et al., 1999; Gielen et al., 2009), with GluN2C-containing receptors $\left(P_{\mathrm{o}} \sim 0.01\right)$ (Dravid et al., 2008) having a $\sim 40$-fold lower $P_{\mathrm{o}}$ than GluN2A-containing receptors $\left(P_{\mathrm{o}} \sim 0.36-0.50\right)$ (Erreger et al., 2004; Gielen et al., 2009). Accordingly, the rate of MK801 current inhibition (see Materials and Methods), as determined by the fit of a single-exponential function to the time course of MK801 inhibition of glutamate-activated current (Fig. $6 C$, dashed overlay), was dramatically slower for GluN1-GluN2C $\left(k=2.68 \times 10^{5} \pm 7000 \mathrm{M}^{-1} \mathrm{~s}^{-1} ; n=8\right)$ than for GluN1GluN2A $\left(k=7.44 \times 10^{6} \pm 1,300,000 \mathrm{M}^{-1} \mathrm{~s}^{-1} ; n=13\right)$ (see below) (Gielen et al., 2009).

We compared the rate of MK801 current inhibition for all GluN1 and GluN2C potentiation positions without (open circle) and after (filled circles) PTrEA (Fig. 6B-D). We did not undertake these and subsequent experiments for two S2-M4 potentiation positions: GluN1(T789C) (Fig. 5A), because its pre-PTrEA current amplitude was small $\left(I_{\text {pre }}<30 \mathrm{nA}\right)$, making it difficult to obtain a reliable measure of the rate of MK801 inhibition; and GluN2C(I793C), because PTrEA-induced current potentiation did not reach steady state (Fig. $5 B$, double asterisks). Additionally, all three M3/M3-S2 potentiation positions, GluN1(A635C), GluN2C(A630C), and GluN2C(E635C), showed significantly reduced steady-state $\mathrm{MK} 801$ inhibition either before and/or after PTrEA application, along with additional inconsistencies, including a notable rebound of current at the end of the MK801 application (supplemental Fig. 2, available at www.jneurosci.org as supplemental material). Because these deviations suggest that MK801 block itself was disrupted (Yuan et al., 2005; Chang and Kuo, 2008), we did not analyze these M3/ M3-S2 potentiation positions further.

For certain positions in S1-M1 and S2M4, the cysteine substitution itself significantly altered the rate of MK801 inhibition from that of wild type $(k \approx$ $\left.2.68 \times 10^{5}\right)$ : GluN1 $(\mathrm{P} 787)$ at S2-M4 $(k=$ $\left.2.72 \times 10^{4} \pm 2000 ; n=6\right)$, GluN2C(S533C) at S1-M1 $\left(6.26 \times 10^{5} \pm\right.$ $85,000 ; n=7)$, and GluN2C(D792C) at S2-M4 $\left(4.95 \times 10^{5} \pm 85,000 ; n=5\right)$ (Fig. $6 C, D$, denoted by asterisks). After PTrEA, all tested GluN1 and GluN2C potentiation positions showed a significant increase in the rate of MK801 inhibition (Fig. 6C,D), suggesting that their open probability $\left(P_{\mathrm{o}}\right)$ was increased. Surprisingly, the relationship between the increase in $P_{\mathrm{o}}$ and the corresponding potentiation of current amplitudes showed a poor correlation $\left(R^{2}=0.37\right)$ when the GluN1 and GluN2C potentiation positions were pooled together (Fig. $6 E$, GluN1 + GluN2C). However, when the GluN1 and GluN2C potentiation positions were considered separately, the resulting correlation drastically improved, with $R^{2}$ values of $\sim 0.8$ for either GluN1 or GluN2C (Fig. $6 E$ ). We do not fully understand this difference between the subunits but it may be a manifestation of subunit-specific contributions to channel gating (Banke and Traynelis, 2003; Blanke and VanDongen, 2008). Nevertheless, the key point here is that the strong correlation within a subunit strongly supports the idea that potentiation is being driven mainly by an increase in $P_{\mathrm{o}}$. The use of MK801 to index a relative $P_{\mathrm{o}}$ is further supported by results with PTrEA-induced inhibition positions (supplemental Fig. 3, available at www. jneurosci.org as supplemental material).

\section{Single-channel activity in a patch increases after PTrEA} exposure to receptors containing potentiation positions Although the MK801 results support the idea that potentiation reflects an increase in $P_{\mathrm{o}}$, there could be some unanticipated systematic effect of PTrEA on the rate of MK801 block. To further verify that PTrEA-induced potentiation is driven by an increase in $P_{\mathrm{o}}$, we measured single-channel activity from outside-out patches isolated from Xenopus oocytes for wild-type receptors and a subset of potentiation positions before and after PTrEA (Fig. 7). We selected one potentiation position in GluN1 S1-M1 (S531), one in GluN2C S1-M1 (V523), and one in GluN2C S2-M4 (S788). Patches certainly contained multiple receptors and we refer to the total time the patch spent in an open state (lower plus 
higher conductances) as the "patch $P_{\mathrm{o}}$ " (see Materials and Methods). For wildtype receptors, the two conductance levels were comparable in amplitude with those reported previously in oocyte patches (Stern et al., 1992), although the mean lifetimes, especially for the higher conductance level, were somewhat longer (supplemental Table 2, available at www.jneurosci. org as supplemental material). As with macroscopic experiments, PTrEA had no notable effects on single-channel currents of wild-type receptors, although there was a slight but insignificant trend toward a reduced patch $P_{\mathrm{o}}\left(P_{\mathrm{o} \text {,post }} / P_{\mathrm{o}}=0.72 \pm 0.04\right.$; $n=3$ ) (Fig. $7 B$, right). The three receptors with cysteine-substituted potentiation positions before PTrEA were indistinguishable from wild-type receptors (supplemental Table 2, available at www.jneurosci.org as supplemental material). In contrast, after PTrEA, all three potentiation positions showed a significant increase in patch activity $\left[P_{\text {o,post }} / P_{\mathrm{o}}\right.$, GluN1 (S531C), $3.64 \pm 0.67$; GluN2C(V523C), $2.13 \pm 0.23$; GluN2C (S788C), $3.02 \pm 0.46 ; n=3$ for each] (Fig. $7 A, B$, right). For GluN2C(V523C) and GluN2C(S788C), PTrEA did not affect the amplitude of either the lower- or higherconductance levels (Fig. $7 B$, left). For GluN1(S531C), however, along with an increase in $P_{\mathrm{o}}$, PTrEA also significantly reduced the amplitude of the higher conductance $\left(\gamma_{\text {post }} / y, 0.91 \pm 0.02\right)$ (Fig. $\left.7 B\right)$. Analysis of open-time distributions before and after PTrEA showed no significant alterations (Fig. 7C), suggesting that the increase in $P_{\mathrm{o}}$ was attributable to a decrease in closed time rather than an increase in open time. Overall, in agreement with the MK801 results, the singlechannel recordings indicate that PTrEA induces a strong increase in open probability, consistent with the idea that current potentiation arises mainly if not exclusively (at least for the three tested positions) from a gating effect of increasing channel $P_{\mathrm{o}}$.

\section{GluN1 potentiation positions show reduced PTrEA-induced current potentiation when coexpressed with GluN2A'}

Wild-type GluN1-GluN2C receptors have a low $P_{\mathrm{o}}(\sim 0.01)$ (Dravid et al., 2008). As judged by MK801 inhibition kinetics, all GluN1-GluN2C cysteine-substituted potentiation positions yield receptors with $P_{\mathrm{o}}$ in a similarly low range (Fig. $6 C, D$ ). After their reaction with PTrEA, all of these potentiation positions showed significant increases in relative $P_{\mathrm{o}}$ (Figs. $6 C, D, 7)$. In contrast, wild-type GluN1-
A

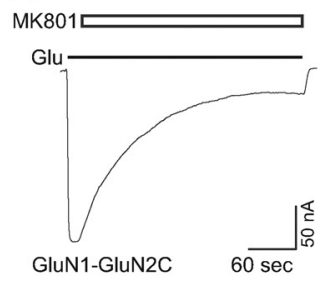

B
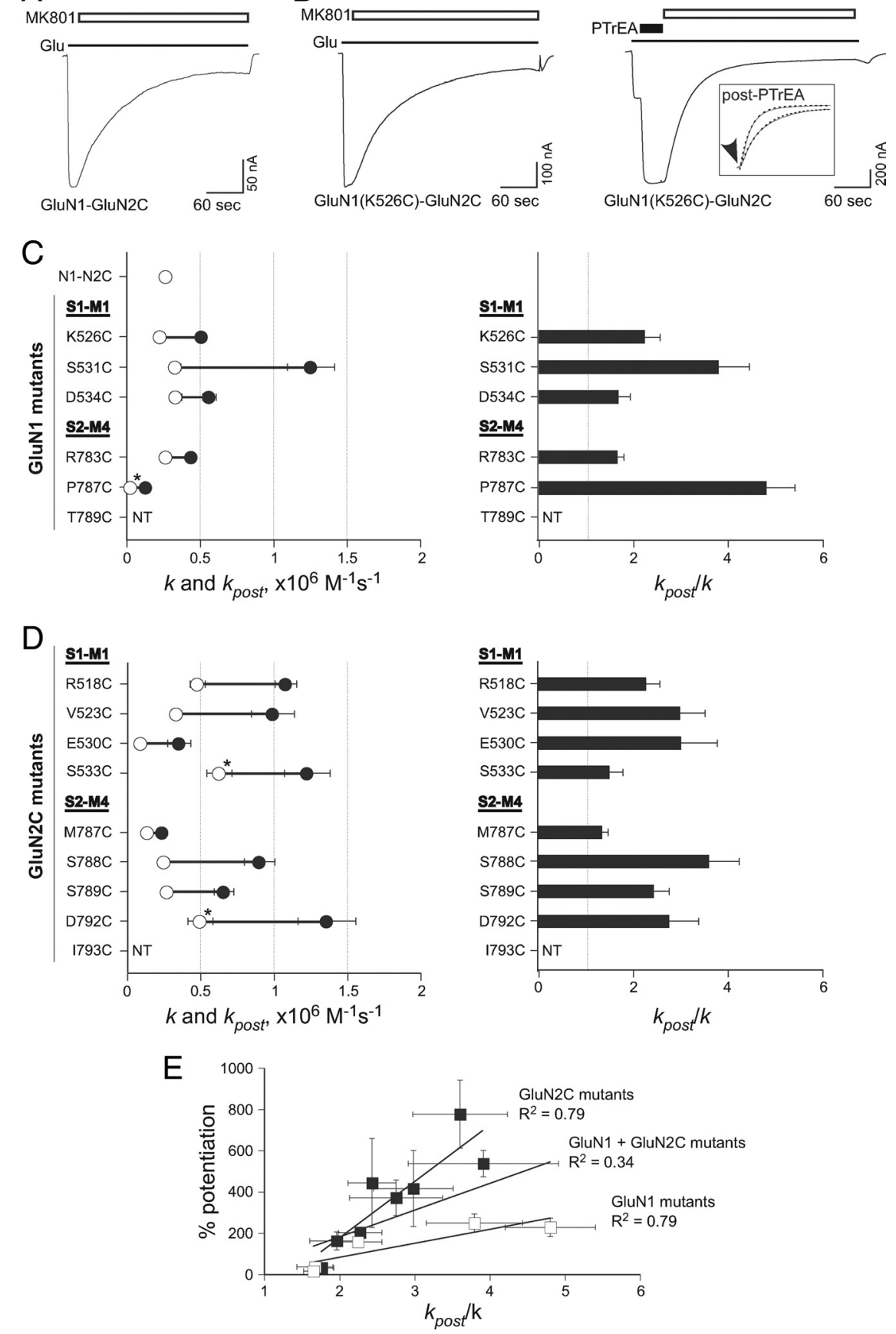

Figure 6. MK801 current inhibition as an index of $P_{0}$. $A$, MK801 inhibition of glutamate-activated currents of wild-type GluN1-GluN2C. After glutamate-activated currents (Glu; thin line) had reached steady state, 50 nм MK801 (unfilled bar) was applied in the presence of agonists until steady-state inhibition was attained. B, MK801 inhibition of glutamate-activated current of GluN1(K526C)-GluN2C without (left) or after (right) PTrEA application (solid bar). When PTrEA was used, MK801 application was initiated once PTrEA-induced potentiation had reached steady state. Inset, The MK801-mediated decays of normalized currents without and after PTrEA (post-PTrEA) were fitted with a single-exponential function (dashed overlays) to determine the time constant $\tau$ of inhibition. The arrowhead indicates onset of MK801 inhibition. C, D, Left, Summary of rate constants of MK801 inhibition without ( $k$, unfilled circles) and after ( $k_{\text {post }}$, filled circles) PTrEA for GluN1 $(\boldsymbol{C})$ and GluN2C (D) potentiation positions. The rate of MK801 inhibition ( $k$ ) of wild-type GluN1-GluN2C is shown in C. Potentiation positions in M3/M3-S2 are not included in this analysis (see text and supplemental Fig. 2, available at www.jneurosci.org as supplemental material). The asterisks denote positions where the cysteine substitution itself significantly altered the rate of MK801 inhibition compared with wild-type GluN1-GluN2C. NT denotes positions not tested because of small current amplitudes [GluN1(S789C)] or incomplete reaction with PTrEA [GluN2C(1793C)]. In some instances, error bars are smaller than the symbols. C, D, Right, The ratio $k_{\text {post }} / k$ for GluN1 (C) and GluN2C (D) potentiation positions. The dotted line indicates $k_{\text {post }} / k=1$. The filled bars indicate positions wherethe rate of MK801 inhibition after PTrEA was significantly different from that without PTrEA $(p<0.05)$. $E$, Correlation between $k_{\text {post }} / k$ and percentage potentiation of whole-cell glutamate-activated current with three linear regressions shown: GluN1 (unfilled squares) and GluN2C (filled squares) positions pooled together (GluN1 + GluN2C) and each subunit considered separately. The $R^{2}$ value for each respective fit is shown. 
A
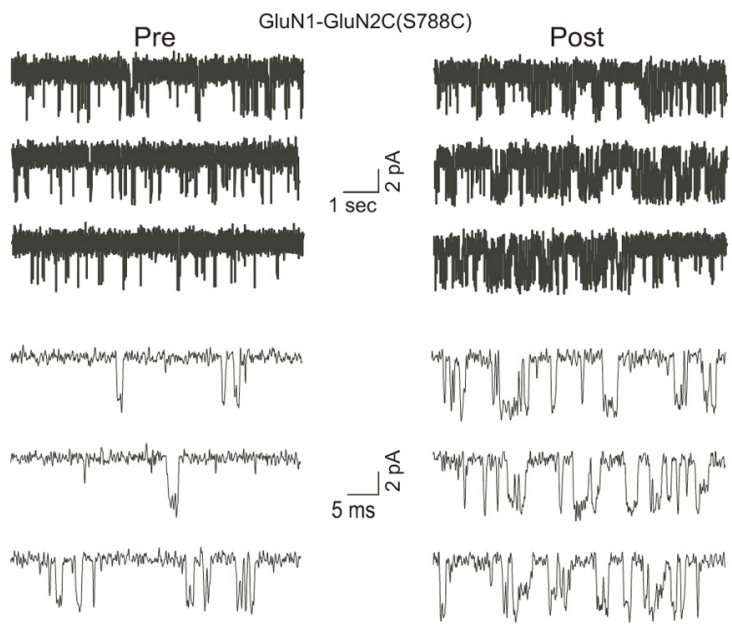

B
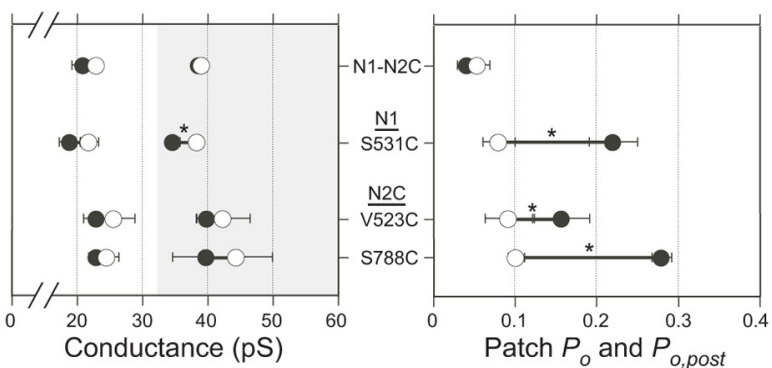

C
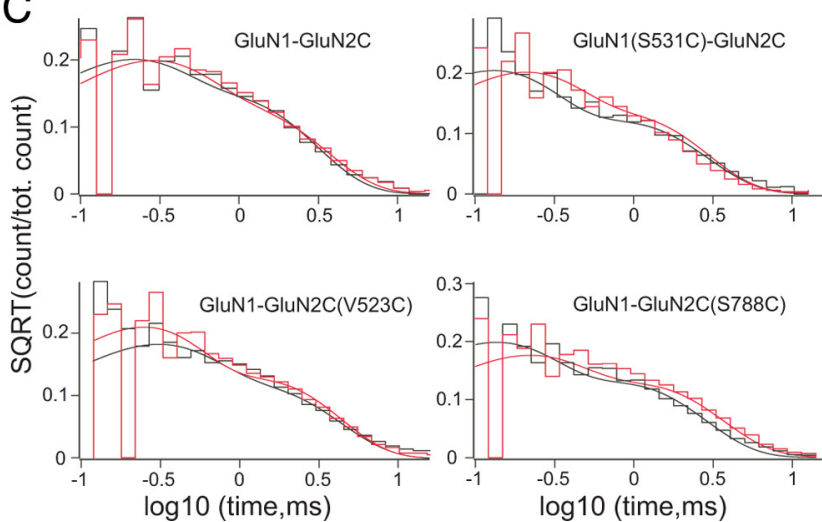

Figure 7. The effect of PTrEA on single-channel currents. $\boldsymbol{A}$, An example outside-out patch from an oocyte injected with GluN1-GluN2C(S788C) showing glutamate-activated singlechannel activity before (pre) and after (post) PTrEA. For display, currents were digitally refiltered at $5 \mathrm{kHz}$ (top) or at $2 \mathrm{kHz}$ (bottom) after being collected at $10 \mathrm{kHz}$. Holding potential was -80 $\mathrm{mV}$. B, Mean values of conductance (left panel) and patch $P_{0}$ (right panel) before (open circles) and after (filled circles) PTrEA ( $n=3$ for each receptor) (see also supplemental Table 2, available at www.jneurosci.org as supplemental material). In the conductance graph, the light and shaded parts show the arbitrary ranges for the lower- and higher-conductance levels, respectively. The asterisks denote statistical significance $(p<0.05)$ in the post/pre ratio from that of the wild-type GluN1-GluN2C. C, Composite open time histograms before (black) and after (red) PTrEA. The data were fit with two exponential components (for values, see supplemental Table 2, available at www.jneurosci.org as supplemental material).

GluN2A inherently has a high $P_{\mathrm{o}}(\sim 0.36-0.50)$ (Erreger et al., 2004), making additional increases in $P_{\mathrm{o}}$ limited (it can increase a maximum of approximately twofold to threefold to reach $P_{\mathrm{o}}=$ 1). If whole-cell current potentiation is a gating effect arising from an increased $P_{\mathrm{o}}$, we reasoned that GluN1 potentiation positions, identified based on coexpression with GluN2C, would show an attenuated degree of PTrEA-induced current potentiation when coexpressed with GluN2A' (a mutant GluN2A subunit where an endogenous cysteine was mutated to alanine) (see Ma- terials and Methods). Figure $8 A-C$ shows the effect of PTrEA on whole-cell currents for the same GluN1-potentation positions coexpressed with either GluN2C (Fig. $8 A, B$, left panels) or GluN2A' (Fig. $8 A, B$, right panels). As expected, PTrEA-induced potentiation for all GluN1 potentiation positions (Fig. $8 C$ ) was significantly reduced when coexpressed with GluN2A' [Fig. $8 \mathrm{~A}$, GluN1(R783C)-GluN2A' $]$ and in some instances was reversed to current inhibition [Fig. 8B, GluN1(S531C)-GluN2A'].

Two GluN1 cysteine-substituted positions, S531C and D534C, showed PTrEA-induced current inhibition when coexpressed with GluN2A' (Fig. 8 B,C). Interestingly, GluN1(S531C), when coexpressed with GluN2C, underwent dual PTrEAinduced effects of increase in $P_{\mathrm{o}}$ and decrease in $\gamma$ (Fig. $7 B$ ). We reasoned that this inhibition of GluN1(cys)-GluN2A' receptors was attributable to a reduction in $\gamma$, an effect masked in GluN2Ccontaining receptors because of their greater increase in $P_{\mathrm{o}}$ driving net whole-cell current potentiation. To test this, we measured the rates of MK801-mediated inhibition of currents in GluN1 (S531C)-GluN2A' and GluN1(D534C)-GluN2A' without (open circles) and after (filled gray circles) reaction to PTrEA (Fig. $8 D, E)$. As a reference, we also measured the rate of MK801 current inhibition for GluN1-GluN2A' (Fig. 8E). After PTrEA, the rate of MK801 current inhibition for GluN1 S531C and D534C, coexpressed with GluN2A', was not significantly altered $\left(k_{\text {post }} / k\right.$ values $=0.98 \pm 0.2$ and $1.63 \pm 0.4$, respectively), in contrast to what occurred when they were coexpressed with GluN2C (Fig. $6 C)$. Thus, the absence of any observable PTrEA-induced changes in $P_{\mathrm{o}}$, as judged from MK801 inhibition kinetics, suggests that a reduction in $\gamma$ underlies whole-cell current inhibition for these two GluN1 positions coexpressed with GluN2A'.

Overall, these results are consistent with PTrEA-induced current potentiation reflecting mainly a gating effect-an increase in $P_{\mathrm{o}}$. Intriguingly, they also suggest that such modulation of NMDAR gating, mediated by the linkers, is dependent on the intrinsic $P_{\mathrm{o}}$ of the receptor, as determined by the expressed GluN2 isoform (GluN2C vs GluN2A). Finally, these results highlight that PTrEA-induced changes in whole-cell currents are the weighted sum of changes in gating $\left(P_{\mathrm{o}}\right)$ and conductance $(\gamma)$.

PTrEA accessibility to the potentiation positions is typically reduced in the channel-closed state

PTrEA-induced potentiation of currents in GluN1-GluN2C cysteine-substituted receptors is driven by an apparent gating effect, but what is its structural basis? Many alternatives could be envisioned including that PTrEA in some way increases the rigidity of a specific linker, increasing the efficacy of coupling the conformational change in the LBD to channel opening. Another possibility, the "closed-state contact" hypothesis, is that potentiation positions are located at points of contact with other structural elements-possibly other linkers, proximal parts of the LBD, transmembrane segments, and/or membrane lipids (Sobolevsky et al., 2007). The bulky PTrEA side chain in such a contact interface would therefore sterically (and/or perhaps electrostatically) limit close (although not necessarily tight) apposition of the contacting partners. If this contact interface is part of an energetically stable closed state, then the energetics of activation gating would be shifted toward channel opening after PTrEA, resulting in an increased $P_{\mathrm{o}}$ and current potentiation.

If the closed-state contact hypothesis is correct, positions at such interfaces should show restricted accessibility in the closed compared with the open state. None of the potentiation positions in and around S1-M1 (Fig. 3) and S2-M4 (Fig. 5) showed discrete binary state dependence to PTrEA, but these steady-state reactivity experi- 
A

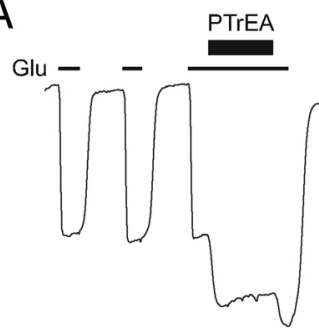

GluN1(R783C)-GluN2C

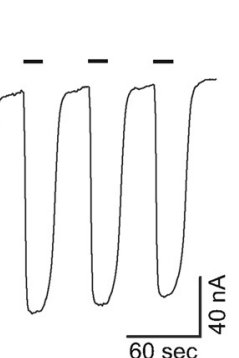

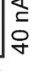

GluN1(R783C)-GluN2A

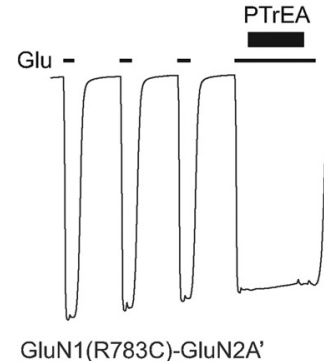

PTrEA

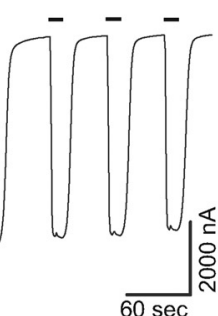

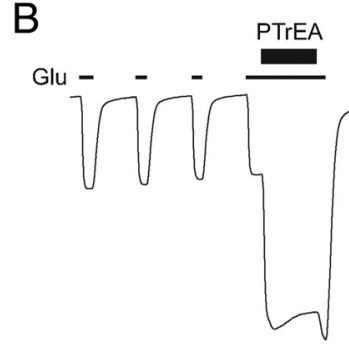

GluN1(S531C)-GluN2C

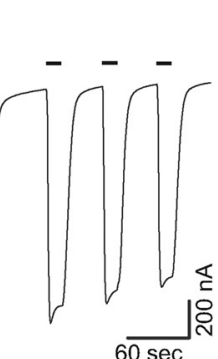

GTu - $+\quad$ PTrEA

Glu -

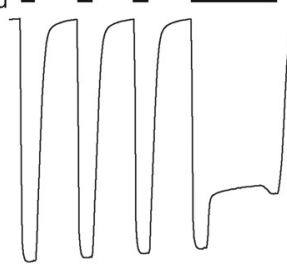

GluN1(S531C)-GluN2A'

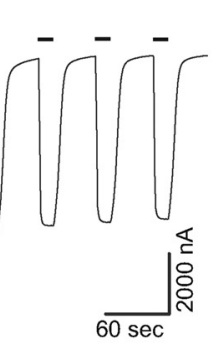

C

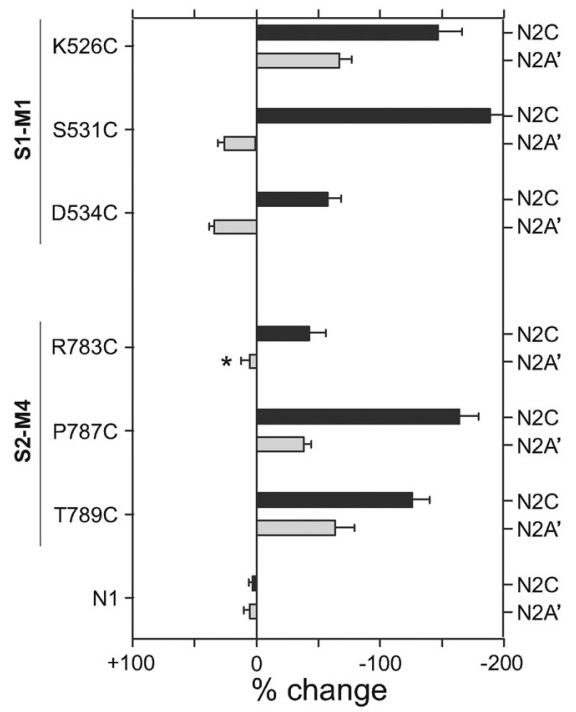

E

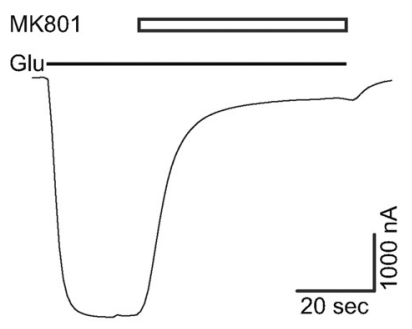

GluN1(S531C)-GluN2A'

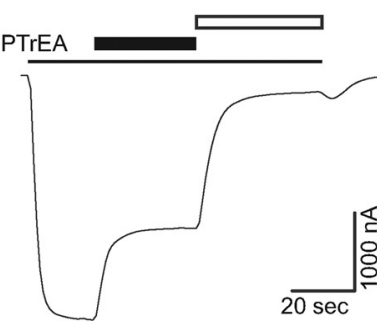

GluN1(S531C)-GluN2A

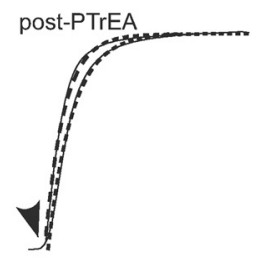

GluN1(S531C)-GluN2A

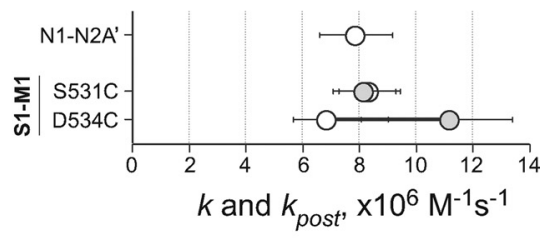

$k$ and $k_{\text {post }}, \times 10^{6} \mathrm{M}^{-1} \mathrm{~s}^{-1}$

Figure 8. Steady-state reaction of PTrEA to GluN1 potentiation positions coexpressed with GluN2C or GluN2A'.A, B, Example recordings showing effects of PTrEA on glutamate-activated current amplitudes for the same GluN1 potentiation positions coexpressed with either GluN2C (left) or GluN2A' (right). The protocol used is identical with that in Figure 2 (see Materials and Methods). For GluN1-GluN2A' NMDARs, we replaced $\mathrm{Ca}^{2+}$ with $\mathrm{Ba}^{2+}$ and added EDTA to the external solution to limit $\mathrm{Ca}^{2+}$-dependent desensitization and $\mathrm{Zn}^{2+}$-mediated modulation, respectively. We recorded a limited number of identical cysteine-substituted GluN1 positions, coexpressed with GluN2C in the $\mathrm{Ba}^{2+}$ - and EDTA-containing solution, and found no significant influence of the differing solutions on PTrEA-induced effects (data not shown). C, Mean percentage change ( \pm 2 SEM) of glutamate-activated current amplitudes after PTrEA for the GluN1 potentiation positions coexpressed with either GluN2C (black bars) or GluN2A' (gray bars). The asterisk denotes percentage change not significantly different from the respective control wild-type GluN1-GluN2C or GluN1-GluN2A' receptors. D, Rate of MK801 current inhibition for GluN1(S531C)-GluN2A'. MK801 (50 nM; unfilled bars) was applied in the continuous presence of agonists (thin black line; Glu) either without (left) or after (middle) PTrEA. The protocols and conditions are the same as in Figure 6B. Single-exponential fits to MK801-mediated decay of normalized currents are shown at right. $E$, Rate constants $k$ (without PTrEA; unfilled circles) and $k_{\text {post }}$ (after PTrEA; filled circles) of MK801 current inhibition for the two GluN1 positions that showed significant PTrEA-induced current inhibition when coexpressed with GluN2A' (from C), as well as for GluN1-GluN2A'.

ments (Figs. 2-5) have low resolution for determining statedependent accessibility. We therefore measured modification rates for PTrEA applied in the presence of agonists, when the channels are open and closed (Fig. 9A, left), or in their absence, when the channels are presumably closed (Fig. 9A, right). For these experiments, we focused on positions that showed $>100 \%$ steady-state potentiation because of the complications of measuring reaction rates for less robust current potentiation. For six of eight tested positions (S531C and P787C in GluN1 and R518C, V523C, S788C, and S789C in GluN2C), modification rates for PTrEA were significantly slower in the absence of agonist $\left(k_{-}\right.$, unfilled circles) than in their presence ( $k_{+}$, filled circles) (Fig. $9 B, C$, left), with $k_{+} / k_{-}$ratios ranging from $\sim 3$ to $\sim 130$ (Fig. $9 B, C$, right). The reduced accessibility in the absence of agonists for these potentiation positions support the notion that they are at contact interfaces in the closed state. For the two other positions, modification rates for PTrEA were either not significantly different [Fig. 9B, GluN1(K526C)] or actually faster [Fig. 9C, GluN2C(E530C)] in the absence than the presence of agonists, suggesting that other mechanisms in addition to the closed-state contact may underlie PTrEA-induced changes in gating.
Degree of whole-cell current potentiation is dependent on the size of the reactive reagent

An additional prediction of the closed-state contact hypothesis is that reagents less bulky than PTrEA would have reduced steric effects and would produce less dramatic increases in $P_{\mathrm{o}}$ and correspondingly would elicit attenuated degrees of current potentiation. We therefore compared the effects of three differently sized (but same valence and general structure) MTS reagents-PTrEA, MTSET, and MTSEA, from large to small, respectively (Fig. $10 A$ ) - on currents for GluN1 and GluN2C potentiation positions. Because this assay is less dependent on initial (pre-MTS) current amplitudes, we were able to test all S1-M1 and S2-M4 potentiation positions. In general, with smaller-sized reagents (i.e., MTSET and MTSEA), the main phenotypes were either an incremental reduction in potentiation [Fig. 10 B, GluN2C(S789C) in S2-M4] or a reversal to net inhibition [Fig. 10C, GluN1(S531C) in S1-M1]. Such size-dependent PTrEA/MTSET/MTSEA patterns occurred for 9 of 13 potentiation positions tested (Fig. 10D, E, positions S531C, D534C, R783C, and P787C in GluN1, and R518C, V523C, S533C, S788C, and S789C in GluN2C) and are consistent 
A

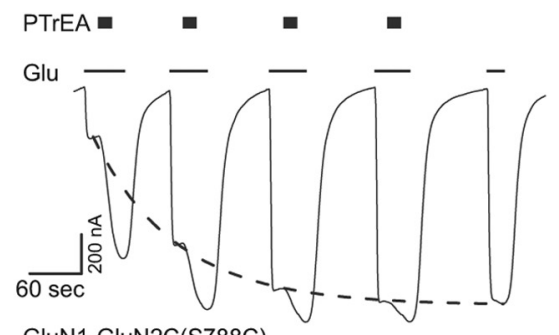

GluN1-GluN2C(S788C)

B

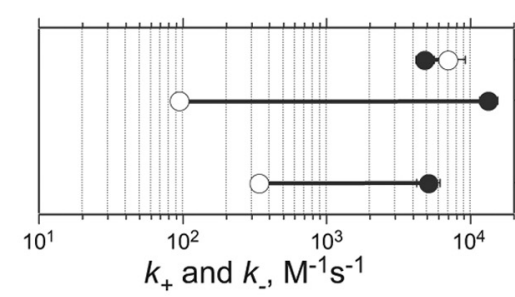

C

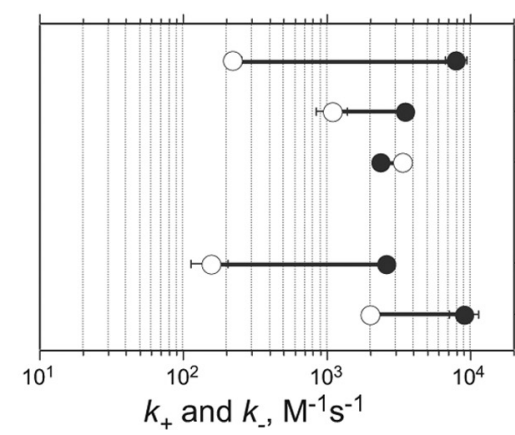

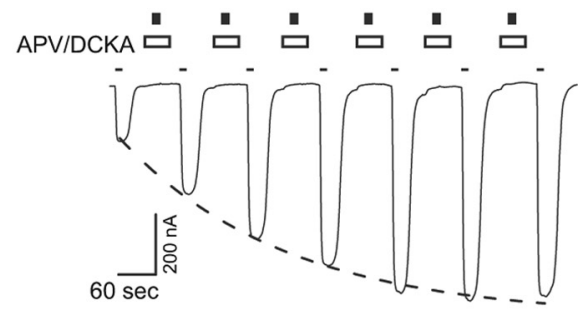

GluN1-GluN2C(S788C)

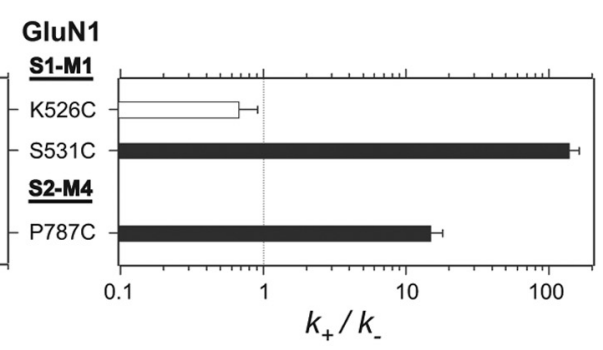

GluN2C

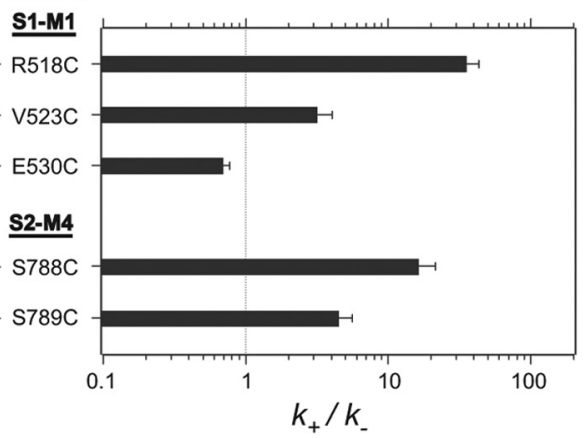

Figure 9. PTrEA modification rates for potentiation positions. A, Pulsive protocol to assay PTrEA (black bars) modification rates of GluN1-GluN2C(S788C) in the presence (left) or absence (right) of agonists (thin line). Left, Twenty-five micromolar PTrEA was applied in the continuous presence of agonists in four $15 \mathrm{~s}$ pulses for a cumulative $60 \mathrm{~s}$ exposure. Right, Two hundred micromolar PTrEA was applied in the absence of agonist, but in the continuous presence antagonists DCKA and APV (open box), in six 15 s pulses for a cumulative $90 \mathrm{~s}$ exposure. The dashed lines denote single-exponential fits of current amplitudes as a function of the cumulative time of PTrEA exposure that defines the rate constants of chemical modification in the presence $\left(k_{+}\right)$or absence $\left(k_{-}\right)$of agonists. $\boldsymbol{B}, \boldsymbol{C}$, Left, Mean values of $k_{+}$(filled circles) and $k_{-}$(open circles) ( \pm 2 SEM) for GluN1 (B) and GluN2C ( $\boldsymbol{C}$ ) potentiation positions. In some instances, error bars are smaller than the symbols. $\boldsymbol{B}, \boldsymbol{C}$, Right, $k_{+} / k_{-}$for GluN1 (B) and GluN2C ( $\boldsymbol{C}$ ) potentiation positions. The filled bars indicate positions where PTrEA modification rates in the presence and absence of agonists were significantly different $(p<0.05)$. The dotted line indicates $k_{+} / k_{-}=1$.

with the idea that these potentiation positions are located at closedstate-dependent contact interfaces. Notably, all six potentiation positions showing reduced PTrEA accessibility in the closed state (Fig. 9) also showed size dependence consistent with the closed-state contact hypothesis (Fig. 10).

Of the remaining four positions, GluN2C(E530C) in S1-M1 showed strong but not significantly different degrees of current potentiation for all three reagents (Fig. 10E), suggesting that charge rather than steric effects may underlie current potentiation. Positions K526C in GluN1 (Fig. 10D) and M787C in GluN2C (Fig. 10E) did not show any size-dependent PTrEA/ MTSET/MTSEA pattern. Additionally, position D792C in GluN2C did show a size-dependent pattern, although it was opposite to our prediction with the degree of current potentiation increasing with larger reagents (Fig. $10 \mathrm{E}$ ). Based on the homologous position in GluA2, it is likely that the membrane environment is confounding MTS accessibility in an unknown manner.

\section{Discussion}

Gating in ionotropic glutamate receptors (GluRs) involves the transduction of structural changes in the LBD initiated by ligand binding/unbinding into physical movements of the ion channel culminating in dilation/constriction of the central pore (Sun et al., 2002; Erreger et al., 2004; Mayer, 2006; Hansen et al., 2007). The transduction machinery between the ligand-binding and transmembrane domains is composed of the linkers S1-M1, M3-S2, and S2-M4. Although M3-S2 is central to the transduction process, our experiments indicate that S1-M1 and S2-M4 can also affect or modulate gating. Furthermore, we identify specific positions in and around S1-M1 and S2-M4 that are important to the energetics of this process and are apparently located at key contact interfaces in the channel-closed state.

\section{PTrEA reaction at potentiation positions in and around S1-M1 and S2-M4 shifts gating equilibrium toward the open state}

Of the 87 positions encompassing the GluN1 and GluN2 linkers tested, 18 of them underwent persistent PTrEA-induced potentiation of whole-cell currents (Figs. 2-5), with 15 of these potentiation positions located in the S1-M1 and S2-M4 linkers. All of the S1-M1 and S2-M4 potentiation positions showed a significantly faster rate of block by the open channel blocker MK-801 after PTrEA than before it (Fig. $6)$, suggesting that current potentiation is attributable to an increase in $P_{\mathrm{o}}$. Confirming this, three potentiation positions [GluN1(S531C), GluN2C(V523C), GluN2C (S788C)] tested with singlechannel recordings all showed a significant increase in $P_{\mathrm{o}}$ after PTrEA (Fig. 7). Thus, PTrEA-induced potentiation of whole-cell currents arises mainly if not exclusively-assuming MK801 is a valid (relative) index of $P_{\mathrm{o}}$-from a gating effect of an increase in the time the receptor spends in the open state, indicating that the dynamics of the S1-M1 and S2-M4 linkers are important components of the NMDAR gating process.

An increase in $P_{\mathrm{o}}$ could arise in one of two ways: either by destabilization of the closed state or stabilization of the open state. Analysis of single-channel recordings of receptors containing potentiation positions showed that, after PTrEA, they had an increased $P_{\mathrm{o}}$ (Fig. $7 A, B$ ) with no significant changes in open time durations (Fig. 7C). Thus, at least for these positions, the increase in $P_{\mathrm{o}}$ most likely arises from a destabilization of the closed state. Additionally, PTrEA-induced potentiation positions were often associated with significant increases in leak currents (supplemental Table 1, available at www.jneurosci.org as supplemental material). Although we did not explore in detail these changes in leak 
A
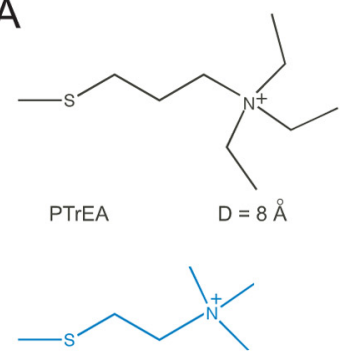

MTSET $D=5.8 \AA$

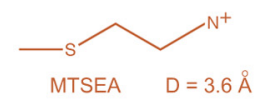

MTSEA $\quad \mathrm{D}=3.6 \AA$
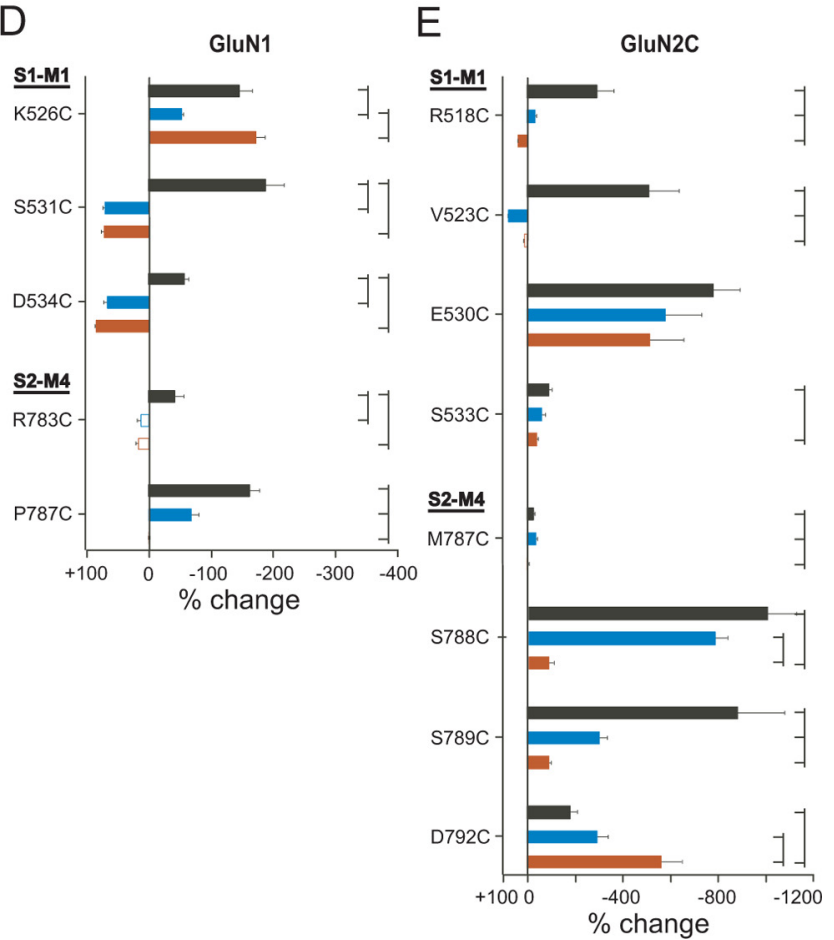

Figure 10. Steady-state reaction of potentiation positions with variably sized MTS reagents. A, Chemical structures of the three differently sized MTS reagents tested: PTrEA (black), MTSET (blue), and MTSEA (brown). The diameters of each side group (Lu et al., 1999) are shown. All reagents have the same valence $(+1)$ and general structure. $\boldsymbol{B}, \boldsymbol{C}$, Glutamate-activated currents for cysteine-substituted GluN1-GluN2C receptors before $\left(I_{\text {pre }}\right)$ and after $\left(I_{\text {post }}\right)$ reaction with MTS reagents: PTrEA, black; MTSET, blue; MTSEA, brown. The protocol used was identical with that shown in Figure 2. For comparison of MTS-induced changes in current amplitude, current amplitudes $\left(I_{\text {pre }}\right)$ before MTS reagent application were normalized. $\boldsymbol{D}, \boldsymbol{E}$, Mean percentage change ( \pm 2SEM) in current amplitude after steady-state exposure of GluN1 (D) or GluN2C (E) potentiation positions to PTrEA (black), MTSET (blue), or MTSEA (brown) in the continuous presence of agonists. The filled bars indicate that the value of percentage change is statistically different from that of wild-type GluN1-GluN2C channels $(p<0.05)$. The symbols to the right of bars indicate statistically significant differences $(p<0.05)$ between the variably sized reagents.

currents, they most likely reflect constitutively open PTrEAreacted NMDARs (Yuan et al., 2005; Schmid et al., 2007; Sobolevsky et al., 2007), presumably because of a destabilized closed state. However, for those potentiation positions not directly tested with single-channel recordings, we cannot rule out that increases in $P_{\mathrm{o}}$ arise from stabilization of the open state.
Structural basis underlying PTrEA-induced increases in $P_{\mathrm{o}}$ The structural basis underlying PTrEA-induced increases in $P_{\mathrm{o}}$ is certainly not the same for all potentiation positions, but identifying these mechanisms will help define elementary gating steps after ligand binding but preceding ion channel opening. One structural mechanism we considered is that certain potentiation positions are located at dynamic contact interfaces in the closed state and that the introduction of a bulky side chain at these positions sterically disrupts the stability of this state, thus shifting gating equilibrium toward the open state (the closed-state contact hypothesis).

Consistent with this hypothesis, we found that most tested potentiation positions showed a reduced degree of access in the closed compared with the open state (Fig. 9). Additionally, the degree of potentiation for 9 of the 13 positions was reduced with decreasing MTS reagent size (Fig. 10), suggesting that steric constraints are important to the gating effect (for a summary of all potentiation positions, see supplemental Table 3 , available at www.jneurosci.org as supplemental material). Notable for these experiments is that positions tested for both state and size dependence showed consistent patterns: either they showed dependencies in both parameters [e.g., GluN1(S531C)] or they showed dependencies in neither parameter [e.g., GluN1(K526C)]. Thus, based on two independent experimental approaches, a subset of the potentiation positions (Fig. 11, red arrowheads) appear to be located at contact interfaces in the channel-closed state. These contact interfaces could be important to receptor function because of specific and local interactions (e.g., electrostatics) between the contacting partners in the closed state or they may be part of the transient statedependent positioning of the overall linkers/transmembrane segments during gating.

Although there is uncertainty in relating specific positions in the NMDAR linkers to those in the AMPAR structure (supplemental Fig. 1, available at www.jneurosci.org as supplemental material), there are some intriguing "hot spots" of potentiation positions. One notable hot spot comprises the adjacent serines in GluN2C S2-M4, S788 and S789 (Fig. 11). In the closed-state AMPAR structure (PDB ID 3KG2), the analogous positions T784 and S785 are located within $4 \AA$ of the extracellular end of the highly conserved SYTANLAAF motif in M3/M3-S2 of an adjacent subunit. This motif contains the "Lurcher" position, substitution of which in NMDARs has strong effects on gating (Jones et al., 2002; Yuan et al., 2005), and the activation gate of the channel (Chang and Kuo, 2008; Sobolevsky et al., 2009). However, reflecting the potential complexity in identifying interactions in the linkers, these S2-M4 positions are also proximal $(<8 \AA)$ to M3/ M3-S2 in the same subunit and M1/S1-M1 in an adjacent subunit. Thus, even with an available structure, identifying gatingdependent contacting partners will be challenging. Nevertheless, identifying them and defining their relative dynamics during gating will be essential in elaborating mechanisms coupling LBD conformational changes to ion channel opening/closing.

Certain positions showed discrete state-dependent accessibility (i.e., apparently accessible in the open but not in the closed state) to PTrEA, suggesting that they are at dynamic contact interfaces, yet PTrEA did not induce current potentiation [Fig. 3B, GluN2C(P531C)]. One possibility is that modification at these positions produced a stronger conductance effect (decrease in $\gamma$ ) outweighing any gating effect (increase in $P_{\mathrm{o}}$ ), thus resulting in net inhibition. Alternatively (or in addition to), the contact interface at these positions may make only minor contributions to the overall energetics of the closed state. This latter alternative is intriguing since it suggests that potentiation positions are specif- 


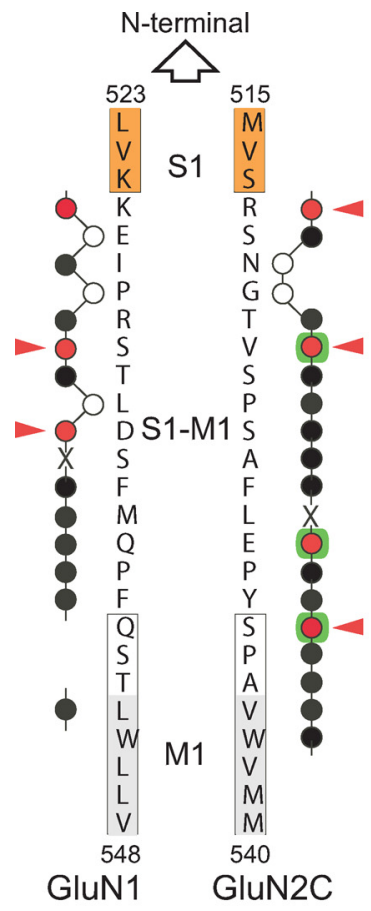

\begin{tabular}{|cl}
\hline & No effect \\
$\mathrm{X}$ & Inhibition \\
$\mathrm{X}$ & Potentiation \\
& No current \\
& $\Delta$ leak
\end{tabular}

Figure 11. Summary of linker positions affecting gating. Summary of discrete effects of PTrEA, applied in the presence of agonists, on substituted cysteines in and around the GluN1 and GluN2C linkers. The adjacent circles indicate that positions were tested for reactivity with PTrEA: unfilled circles, no effect on glutamate-activated current; black circles, current inhibition; red circles, current potentiation. X, No detectable glutamate-activated current; green highlight, significant PTrEA-induced change in leak current (from Figs. 3C, 4C,5C). Also highlighted here are the nine potentiation positions proposed to be at transient contact interface in the closed state (red arrowheads).

ically identifying linker elements (or structures) that are critical to gating.

\section{PTrEA-induced gating effects are specific to the GluN2 subunit and dependent on the intrinsic $P_{\mathrm{o}}$ of the NMDAR} GluN1 potentiation positions, defined by coexpression with GluN2C, showed reduced degrees of PTrEA-induced current potentiation when coexpressed with GluN2A' (Fig. 8). NMDAR $P_{\mathrm{o}}$ is GluN2 subunit specific (Chen et al., 1999; Gielen et al., 2009; Yuan et al., 2009). GluN2A-containing NMDARs have a relatively high intrinsic $P_{\mathrm{o}}(0.36-0.50)$ (Erreger et al., 2004), whereas for GluN2C-containing NMDARs it is much lower $(\sim 0.01)$ (Dravid et al., 2008). Hence, for GluN2A-containing receptors, the energetics of channel gating are already shifted more toward the open state than for GluN2C-containing receptors, limiting the potential for additional increases. In contrast, for GluN2Ccontaining receptors, where channel opening is intrinsically disfavored, there exists greater energetic "room" to increase $P_{\mathrm{o}}$. Thus, the inherent biophysical gating properties of NMDARs dictate the directionality and extent of linker-mediated modulation of gating.

\section{Novel sites and mechanism of modulating NMDAR function} NMDARs are implicated in diverse neurological and psychiatric diseases (Kalia et al., 2008). However, since they are ubiquitous in the CNS and subserve numerous physiological functions, selec- tive targeting of dysfunctional NMDARs is essential for any clinically useful drug. Indeed, competitive antagonists at either the GluN1 glycine-binding site or the GluN2 glutamate-binding site have been abandoned in clinical trials (Kalia et al., 2008) mainly because of their indiscriminate actions on all NMDARs. In contrast, allosteric modulators at the $\mathrm{N}$-terminal domain, such as the GluN2B-specific ifenprodil, show promise in subunitspecific targeting, although their clinical utility is yet unknown (Paoletti and Neyton, 2007). At the ion channel, several classes of pore blockers are used for limited purposes (e.g., ketamine for anesthesia and memantine for moderateto-severe Alzheimer's disease) (Lipton, 2006).

Here, we identify the NMDAR linker region as a novel pharmacologic target wherein drug action, in a noncompetitive mechanism, could modulate gating. The linkers are extracellularly exposed, as evidenced from their wide accessibility to PTrEA (Figs. 3-5). More specifically, targeting of S1-M1 and S2-M4, which are remote from the central axis of the pore and do not directly control channel gating (unlike M3-S2), may offer more subtle means of modulating NMDAR function, potentially lessening side effects. Finally, since the proposed mechanism of drug action at the linkers regulates gating energetics, any modulation of receptor activity is dictated by the inherent gating properties of the NMDAR, which themselves are highly dependent on the specific GluN2 subunit. This concept can be used to attain subunit specificity in drug action and exploit the spatiotemporal localization of the different GluN2 subunits in the CNS. Hence, NMDAR activity can be strongly potentiated for GluN1-GluN2C, whereas it can be strongly inhibited for GluN1-GluN2A. Intriguingly, memantine and amantadine, two drugs currently in clinical use and known to function as pore blockers, might also have gating effects by interacting with the linkers as secondary sites of action (Blanpied et al., 2005; Chen and Lipton, 2005; Kotermanski et al., 2009).

\section{References}

Balannik V, Menniti FS, Paternain AV, Lerma J, Stern-Bach Y (2005) Molecular mechanism of AMPA receptor noncompetitive antagonism. Neuron 48:279-288.

Banke TG, Traynelis SF (2003) Activation of NR1/NR2B NMDA receptors. Nat Neurosci 6:144-152.

Beck C, Wollmuth LP, Seeburg PH, Sakmann B, Kuner T (1999) NMDAR channel segments forming the extracellular vestibule inferred from the accessibility of substituted cysteines. Neuron 22:559-570.

Blanke ML, VanDongen AM (2008) The NR1 M3 domain mediates allosteric coupling in the $N$-methyl-D-aspartate receptor. Mol Pharmacol 74:454-465.

Blanpied TA, Clarke RJ, Johnson JW (2005) Amantadine inhibits NMDA receptors by accelerating channel closure during channel block. J Neurosci 25:3312-3322.

Chang HR, Kuo CC (2008) The activation gate and gating mechanism of the NMDA receptor. J Neurosci 28:1546-1556. 
Chen HS, Lipton SA (2005) Pharmacological implications of two distinct mechanisms of interaction of memantine with $N$-methyl-D-aspartategated channels. J Pharmacol Exp Ther 314:961-971.

Chen N, Luo T, Raymond LA (1999) Subtype-dependence of NMDA receptor channel open probability. J Neurosci 19:6844-6854.

Choi Y, Chen HV, Lipton SA (2001) Three pairs of cysteine residues mediate both redox and $\mathrm{Zn}^{2+}$ modulation of the NMDA receptor. J Neurosci 21:392-400.

Cull-Candy SG, Leszkiewicz DN (2004) Role of distinct NMDA receptor subtypes at central synapses. Sci STKE 2004:re16.

Dilmore JG, Johnson JW (1998) Open channel block and alteration of $N$-methyl-D-aspartic acid receptor gating by an analog of phencyclidine. Biophys J 75:1801-1816.

Dravid SM, Erreger K, Yuan H, Nicholson K, Le P, Lyuboslavsky P, Almonte A, Murray E, Mosely C, Barber J, French A, Balster R, Murray TF, Traynelis SF (2007) Subunit-specific mechanisms and proton sensitivity of NMDA receptor channel block. J Physiol 581:107-128.

Dravid SM, Prakash A, Traynelis SF (2008) Activation of recombinant NR1/ NR2C NMDA receptors. J Physiol 586:4425-4439.

Erreger K, Chen PE, Wyllie DJ, Traynelis SF (2004) Glutamate receptor gating. Crit Rev Neurobiol 16:187-224.

Gielen M, Siegler Retchless B, Mony L, Johnson JW, Paoletti P (2009) Mechanism of differential control of NMDA receptor activity by NR2 subunits. Nature 459:703-707.

Hansen KB, Yuan H, Traynelis SF (2007) Structural aspects of AMPA receptor activation, desensitization and deactivation. Curr Opin Neurobiol 17:281-288.

Huettner JE, Bean BP (1988) Block of N-methyl-D-aspartate-activated current by the anticonvulsant MK-801: selective binding to open channels. Proc Natl Acad Sci U S A 85:1307-1311.

Jahr CE (1992) High probability opening of NMDA receptor channels by L-glutamate. Science 255:470-472.

Jones KS, VanDongen HM, VanDongen AM (2002) The NMDA receptor M3 segment is a conserved transduction element coupling ligand binding to channel opening. J Neurosci 22:2044-2053.

Kalia LV, Kalia SK, Salter MW (2008) NMDA receptors in clinical neurology: excitatory times ahead. Lancet Neurol 7:742-755.

Kotermanski SE, Wood JT, Johnson JW (2009) Memantine binding to a superficial site on NMDA receptors contributes to partial trapping. J Physiol 587:4589-4604.

Krupp JJ, Vissel B, Heinemann SF, Westbrook GL (1996) Calciumdependent inactivation of recombinant $N$-methyl-D-aspartate receptors is NR2 subunit specific. Mol Pharmacol 50:1680-1688.

Krupp JJ, Vissel B, Heinemann SF, Westbrook GL (1998) N-terminal domains in the NR2 subunit control desensitization of NMDA receptors. Neuron 20:317-327.

Krystal JH, D’Souza DC, Mathalon D, Perry E, Belger A, Hoffman R (2003) NMDA receptor antagonist effects, cortical glutamatergic function, and schizophrenia: toward a paradigm shift in medication development. Psychopharmacology (Berl) 169:215-233.

Lipton SA (2006) Paradigm shift in neuroprotection by NMDA receptor blockade: memantine and beyond. Nat Rev Drug Discov 5:160-170.

Lu T, Nguyen B, Zhang X, Yang J (1999) Architecture of a $\mathrm{K}^{+}$channel inner pore revealed by stoichiometric covalent modification. Neuron 22:571-580.

Mayer ML (2005) Glutamate receptor ion channels. Curr Opin Neurobiol 15:282-288.

Mayer ML (2006) Glutamate receptors at atomic resolution. Nature 440: $456-462$.

Oswald RE, Ahmed A, Fenwick MK, Loh AP (2007) Structure of glutamate receptors. Curr Drug Targets 8:573-582.

Paoletti P, Neyton J (2007) NMDA receptor subunits: function and pharmacology. Curr Opin Pharmacol 7:39-47.

Qin F (2004) Restoration of single-channel currents using the segmental $k$-means method based on hidden Markov modeling. Biophys J 86: $1488-1501$.

Rosenmund C, Feltz A, Westbrook GL (1995) Synaptic NMDA receptor channels have a low open probability. J Neurosci 15:2788-2795.

Schmid SM, Körber C, Herrmann S, Werner M, Hollmann M (2007) A domain linking the AMPA receptor agonist binding site to the ion pore controls gating and causes lurcher properties when mutated. J Neurosci 27:12230-12241.

Sobolevsky AI, Beck C, Wollmuth LP (2002) Molecular rearrangements of the extracellular vestibule in NMDAR channels during gating. Neuron 33:75-85.

Sobolevsky AI, Prodromou ML, Yelshansky MV, Wollmuth LP (2007) Subunit-specific contribution of pore-forming domains to NMDA receptor channel structure and gating. J Gen Physiol 129:509-525.

Sobolevsky AI, Rosconi MP, Gouaux E (2009) X-ray structure, symmetry and mechanism of an AMPA-subtype glutamate receptor. Nature 462:745-756.

Stern P, Béhé P, Schoepfer R, Colquhoun D (1992) Single-channel conductances of NMDA receptors expressed from cloned cDNAs: comparison with native receptors. Proc Biol Sci 250:271-277.

Südhof TC, Malenka RC (2008) Understanding synapses: past, present, and future. Neuron 60:469-476.

Sun Y, Olson R, Horning M, Armstrong N, Mayer M, Gouaux E (2002) Mechanism of glutamate receptor desensitization. Nature 417:245-253.

Villarroel A, Regalado MP, Lerma J (1998) Glycine-independent NMDA receptor desensitization: localization of structural determinants. Neuron 20:329-339.

Watanabe J, Beck C, Kuner T, Premkumar LS, Wollmuth LP (2002) DRPEER: a motif in the extracellular vestibule conferring high $\mathrm{Ca}^{2+}$ flux rates in NMDA receptor channels. J Neurosci 22:10209-10216.

Waxman EA, Lynch DR (2005) N-Methyl-D-aspartate receptor subtypes: multiple roles in excitotoxicity and neurological disease. Neuroscientist 11:37-49.

Yelshansky MV, Sobolevsky AI, Jatzke C, Wollmuth LP (2004) Block of AMPA receptor desensitization by a point mutation outside the ligandbinding domain. J Neurosci 24:4728-4736.

Yuan H, Erreger K, Dravid SM, Traynelis SF (2005) Conserved structural and functional control of $N$-methyl-D-aspartate receptor gating by transmembrane domain M3. J Biol Chem 280:29708-29716.

Yuan H, Hansen KB, Vance KM, Ogden KK, Traynelis SF (2009) Control of NMDA receptor function by the NR2 subunit amino-terminal domain. J Neurosci 29:12045-12058. 